\title{
Computerized Heart Sounds Analysis
}

\author{
S.M. Debbal \\ Genie -Biomedical Laboratory (GBM), \\ Department of electronic \\ Faculty of science engineering \\ university Aboubekr Belkaid \\ Algeria
}

\section{Introduction}

Heartbeat sound analysis by auscultation is still insufficient to diagnose some heart diseases. It does not enable the analyst to obtain both qualitative and quantitative characteristics of the phonocardiogram signals [1],[2]. Abnormal heartbeat sounds may contain, in addition to the first and second sounds, S1 and S2, murmurs and aberrations caused by different pathological conditions of the cardiovascular system [2]. Moreover, in studying the physical characteristics of heart sounds and human hearing, it is seen that the human ear is poorly suited for cardiac auscultation [3]. Therefore, clinic capabilities to diagnose heart sounds are limited.

The sound emitted by a human heart during a single cardiac cycle consist of two dominant events, known as the first heart sound S1 and the second heart sound S2 (Figure1), S1 relates to the closing of the mitral and tricuspid valves whilst S2 is generated by the halting of the aortic and pulmonary valves leaflets [1]. S1 corresponds in timing to the QRS complex in ECG (Electrocardiogram) and S2 follows the systolic pause in the normal cardiac cycle. Heartbeat sound analysis by auscultation only is still insufficient to diagnose some heart diseases. It does not enable the analyst to obtain both qualitative and quantitative characteristics of S1 and S2 of the phonocardiogram [1-2]. Moreover, in studying the physical characteristics of heart sounds and human hearing, it is seen that the human ear is poorly suited for cardiac auscultation [3]. Therefore, clinic capabilities to diagnose heart sounds are limited. In this paper we are interested in the study of the physical characteristics of the second heart sound 52 which consist of two major components in the spectrum of the signal. One of these components A2 is due to the closure of the aortic valve and the other $\mathrm{P} 2$ is due to the closure of the pulmonary valve.

The aortic component is loudest than the pulmonic component. It is discernible at all the auscultation sites.It is best heard at the right base, with the diaphragm of the chest piece firmly pressed, whereas the pulmonic on a may only be audible at the left base, with the diaphragm of the chest piece firmly pressed.

The aortic component has higher frequency contents and generally precedes the pulmonary component because in normal heart activity the aortic valve closes before the pulmonary valve. The difference of time occurrence between these valves activities is known in medical 
community [4 - 6] as split. However in many diseases [1] this order of time occurrence may be reverse and its delay varies.

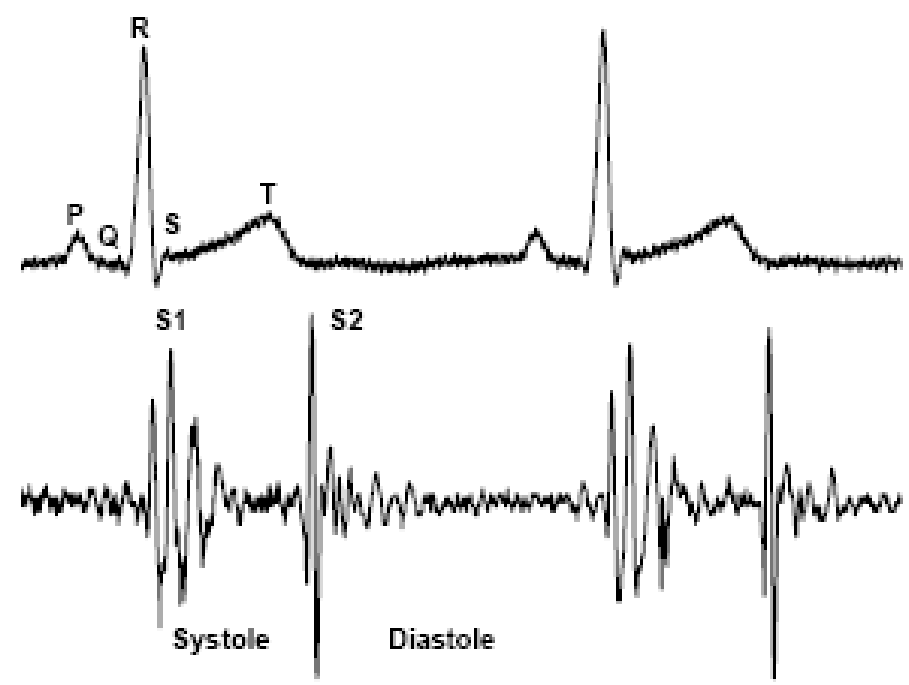

Fig. 1. Correlation between the phonocardiogram signal (PCG) and the electrocardiogram signal (ECG)

The characteristics of the PCG signal and other features such as heart sounds S1 and S2 location; the number of components for each sound; their frequency content; their time interval; all can be measured more accurately by digital signal processing techniques.

The FFT (Fast Fourier Transform) can provide a basic understanding of the frequency contents of the heart sounds. However, FFT analysis remains of limited values if the stationary assumption of the signal is violated. Since heart sounds exhibit marked changes with time and frequency, they are therefore classified as non - stationary signals. To understand the exact feature of such signals, it is thus important, to study their time frequency characteristics.

In this paper the wavelet transform is used to analyse both the normal and abnormal heart sound in both time and frequency domains. This technique has been shown to have a very good time resolution for high-frequency components. In fact the time resolution increases as the frequency increases and the frequency resolution increases as the frequency decreases [4],[5].

Furthermore, the wavelet transform has demonstrated the ability to analyse the heart sound more accurately than other techniques STFT or Wigner distribution [6] in some pathological cases.

In fact the spectrogram (STFT) cannot track very sensitive sudden changes in the time direction. To deal with these time changes properly it is necessary to keep the length of the time window as short as possible. This however, will reduce the frequency resolution in the 
time-frequency plane. Hence, there is a trade-off between time and frequency resolutions [6].

However the Wigner distribution (WD) and the corresponding WVD (Wigner Ville Distribution) have shown good performances in the analysis of non-stationary signals. This comes from the ability of the WD to separate signals in both time and frequency directions. One advantage of the WD over the STFT is that it does not suffer from the time-frequency trade-off problem. On the other hand, the WD has a disadvantage since it shows crossterms in its response. These cross-terms are due to the nonlinear behaviour of the WD, and bear no physical meaning. One way to remove these cross-terms is by smoothing the timefrequency plane, but this will be at the expense of decreased resolution in both time and frequency [7].

The WD was applied to heart sound signal it shows no succes in displaying or separating the signal components in both the time and frequency direction [6], although it provides high time-and frequency- resolution in simple monocomponent signal analysis[8].

To overcome these difficulties with the STFT and the WD an alternative way to analyse the non-stationary signals is the wavelet transform (WT). It expand the signal some basis functions. The basis functions can be constructed by dilation, contractions and shifts of a unique function called the "wavelet prototype" or "wavelet mother". The WT act as "mathematical microscope" in which we can observe different parts of the signal by just adjusting the focus.

The wavelet Transform is a technique in the domain of time-frequency distributions. The main idea of this method is the representation of an arbitrary signal as a superposition of basic signals, "atoms", located in time and frequency. These atoms may be derived by means of a special operation on a single parent atom. Parent atoms and derivation operation are usually chosen such as to enable the construction of an orthonormal system [9].

The study of the decomposition of the signal in " atoms " was first carried out by Gabor however, it was quickly abandoned be cause of :

1. the no simultaneous Representation in time and frequency

2. grid made up of rectangular cells is not a flexible device

3. the mathematical theory of the phenomenon is badly structured.

The representation time-scale of WT based on a dyadic paving appears more flexible. It a mathematical structure governed by a formula of exact inversion [10] making possible the existence of orthonormal basis. This makes the wavelet to be a simultaneous function of time and frequency.

In this paper the continuous wavelet transform (CWT) is applied to analyse pathological PCG signals. The CWT is more appropriate than the discrete wavelet transform (DWT), since we are interested in the analysis of non-stationary signals and not signal coding where DWT is found to be more useful

\section{Theoretical background}

\subsection{Fourier Transform (FT)}

In 1882, Joseph Fourier discovered that any periodic function could be represented as an infinite sum of periodic complex exponential functions [10]. The inclusive property of only periodic functions was later extended to any discrete time function. The Fourier transform (FT) [as regular Fourier Transform] converts a signal expressed in the time domain to a signal expressed in the frequency domain. The FT is widely used and usually implemented 
in the form of FFT algorithm (fast Fourier transform). The mathematical definition of the FT is given below.

$$
X(f)=\int x(t) e^{-j 2 \Pi f t} d t
$$

Where $\mathrm{t}$ and $f$ are respectively the time and frequency parameters. The time domain signal $x(t)$ is multiplied by a complex exponential at a frequency $f$ and integrate over all time. In other words, any discrete time signal may be represented by a sum of sines and cosines, which are shifted and are multiplied by a coefficient that changes their amplitude. $X(f)$ are the Fourier coefficients which are large when a signal contains a frequency component around the frequency $f$.

The peaks in a plot of the FT of a signal correspond to dominant frequency components of the signal. Fourier analysis is simply not effective when used on non stationary signals because it does not provide frequency content information localized in time. Most real world signals exhibit non stationary characteristics (such as heart sound signals). Thus, Fourier analysis is not adequate.

\subsection{Short-time Fourier Transform (STFT)}

The STFT is obtained by calculating the Fourier transform of a sliding windowed version of the time signal $\mathrm{s}(\mathrm{t})$. The location of the sliding window adds a time dimension and one gets a time-varying frequency analysis.

The mathematical representation of STFT is :

$$
S(t, f)=\int_{-\infty}^{+\infty} s(\tau) w(\tau-t) e^{-j 2 \pi f t} d \tau
$$

Where $w(\tau-t)$ it is the sliding window applied to the signal $s(t), f$ is the frequency and $t$ is the time.

The length of the window is chosen so that to maintain signal stationary in order to calculate the Fourier transform. To reduce the effect of leakage (the effect of having finite duration), each sub-record is then multiplied by an appropriate window and then the Fourier transform is applied to each sub-record. As long as each sub-record does not contain rapid changes the spectrogram will give an excellent idea of how the spectral composition of the signal has changed during the whole time record. However, there exist many physical signals whose spectral content is so rapidly changing that finding an appropriate short-time window is problematic, since there may not be any time interval for which the signal is stationary. To deal with these time changes properly it is necessary to keep the length of the time window as short as possible. This, however, will reduce the frequency resolution in the time-frequency plane. Hence, there is a trade-off between time and frequency resolutions.

\subsection{Wgner Distribution function (WD)}

In contrast to the STFT, which is resolution limited either in time or in frequency (dictated by the window function), and suffers from smearing and side lobe leakage, the WD offers excellent resolution in both the frequency and time domains. The WD of two signals, $x(t)$, $y(t)$, is defined via, 


$$
W(t, \omega)=\int_{-\infty}^{+\infty} x\left(t+\frac{\tau}{2}\right) y^{*}\left(t-\frac{\tau}{2}\right) e^{-j \omega \tau} d \tau
$$

The auto WD is obtained when $x(t)=y(t)=\mathrm{s}(\mathrm{t})$, it is a bilinear function of the signal $\mathrm{s}(t)$. the $\mathrm{WD}$, and can also be expressed as

$$
S(t, \omega)=\int_{-\infty}^{+\infty} s\left(t+\frac{\tau}{2}\right) s^{*}\left(t-\frac{\tau}{2}\right) e^{-j \omega \tau} d \tau
$$

Where $\mathrm{t}$ and $\omega$ are respectively, the time and frequency variables, and * denotes the complex conjugate.

The WD had shown good applications in the analysis of non-stationary signal [7]. This comes from the ability of this method to separate signals in both time and frequency directions. The WD has a disadvantage that it is limited by the appearance of cross-terms. These cross-terms are due to the nonlinear behaviour of the WD, and bear no physical meaning. One way to remove these cross-terms is by smoothing the time-frequency plane [7], but this will be at the expense of decreased resolution in both time and frequency.

\subsection{Continuous Wavelet Transform (CWT)}

The continuous wavelet transform was developed as a method to obtain simultaneous, high resolution time and frequency information about a signal. The term 'wavelet' was first mentioned in 1909 in a thesis by Alfred Haar [M.Misiti.Y.Misiti.G.Oppenheim.J.M.Poggi.Wavelet Toolbox:For use with MATLAB.The Math Works Inc.1996], although the progress in the field of wavelet has been relatively slow until the 1980's when scientist and engineers from different fields realized they were working on the same concept and began collaborating .

The CWT rather than the STFT uses a variable sized window region .Because the wavelet may be dilated or compressed, different features of the signal are extracted. While a narrow wavelet extracts high frequency components, a stretched wavelet picks up on the lower frequency components of the signal.

The CWT is computed by correlating the signal $s(t)$ with families of time-frequency atoms $\mathrm{g}(\mathrm{t})$, it produce a set of coefficients $\mathrm{C}(\mathrm{a}, \mathrm{b})$ given by :

$$
\begin{gathered}
C(a, b)=\frac{1}{\sqrt{a}} \int_{-\infty}^{+\infty} s(t) g *\left(\frac{t-b}{a}\right) d t \\
=\sqrt{a} \int_{-\infty}^{+\infty} G(a w) S(w) e^{j b w} d w
\end{gathered}
$$

Where

- $\quad \mathrm{b}$ is the time location

- $\quad \mathrm{a}$ is called scale factor and it is inversely proportional to the frequency $(\mathrm{a}>0)$

- *dénotes a complexe conjugate.

- $\mathrm{g}(\mathrm{t})$ is the analysing wavelet .

- $S(w)$ and $G(w)$ are, respectively, the Fourier transforms of $s(t)$ and $g(t)$. 
The analyzing wavelet function $\mathrm{g}(\mathrm{t})$ should satisfy some properties. The most important ones are continuity, integrability, square integrability, progressivity and it has no d.c component. Moreover, the wavelet $\mathrm{g}(\mathrm{t})$ has to be concentrated in both time and frequency as much as possible. It is well known that the smallest time-bandwidth product is achieved by the Gaussian function [3]. Hence the most suitable analyzing wavelet for time-frequency analysis is the complex exponential modulated Gaussian function of the form :

$$
g(t)=\exp \left(-\frac{t^{2}}{2}+j w_{O} t\right)
$$

If we choose the analyzing wavelet that has the following Fourier transform (FT):

$$
\mathrm{G}(w)=\operatorname{Aexp}\left[-\left(w-w_{0}\right)^{2} / 2\right]+\varepsilon
$$

When $\varepsilon$ is a small correction term, theoretically necessary to satisfy the admissibility conditions of wavelets; $w_{0}$ is chosen large enough so that the correction term is negligible and can be ignored.

This is known as the Gabor wavelet. It was shown [5] that $w_{O}=5.33$, which is enough to make the correction term negligible and gives an optimal time-bandwidth product.

In a continuous wavelet transform, the wavelet corresponding to the scale and the time location $b$ is given by

$$
g_{a, b}(\mathrm{t})=1 / \sqrt{|a|} \mathrm{g}\left(\frac{t-b}{a}\right)
$$

Where $\mathrm{g}(\mathrm{t})$ is the wavelet "prototype" or mother which can be thought of as a band pass function. The factor $/ a /^{-1 / 2}$ is used to ensure energy preservation [5].

\subsection{Discrete Wavelet Transform (DWT)}

Wavelet series (WS) coefficients are sampled CWT coefficients. Time remains continuous but time-scale parameters $(b, a)$ are sampled on a so-called "dyadic" grid in the time-scale plane $(b, a)[11]$. A common definition is :

$$
C_{j k}=\operatorname{CWT}\left\{\mathrm{s}(\mathrm{t}) ; \mathrm{a}=2^{j}, \mathrm{~b}=\mathrm{k} 2^{j}\right\} \text { with } \mathrm{j}, \mathrm{k} \in \mathrm{Z}
$$

The wavelets are in this case :

$$
\Psi_{\mathrm{jk}}(\mathrm{t})=2^{-j / 2} \quad \Psi\left(2^{-j} \mathrm{t}-\mathrm{k}\right)
$$

The discrete wavelet transform (DWT) has been recognized as a natural wavelet transform for discrete-time signals. Both the time and time-scale parameters are discrete.

The discretization process partially depends upon the algorithm chosen to perform the transformation.

The $C_{j, k}$ could be well approximated by digital filter banks. By using Mallat's [12] remarkable fast pyramid algorithms which involve use of low-pass and high-pass filters. The Mallat algorithm is in fact a classical scheme known in the signal processing community as two-channel subband coder. The wavelet analysis permits to decompose the 
phonocardiogram signal in filter banks. The signal to be analyzed is decomposed in approximation an in detail while using two filter $\mathrm{h}$ and $\mathrm{g}$. The $\mathrm{h}$ filter is a low - pass filter with a band pass $[0, \Pi / 2]$, it generates the approximation signal " $\mathrm{A}$ ".

The $g$ filter is a high - pass filter of band pass $[0, \Pi]$, it generates the detail signal " $D "$. The filtered signals are under-sampled (decimation) according to the rule of Nyquist. This leads to a considerable reduction in computing time. Every approximation is decomposed all over again in approximation and detail. In this case the signal is decomposed into several frequency bands instead of two bands. The number of bands depends on decomposition level.

Figure2 shows the discrete wavelet decomposition on three levels, as well as the note associated filter bank for each level. We note that the width of the filter banks band-pass is veritable according to the decomposition level. The wavelet analysis permits to decompose the phonocardiogram signal in filter banks. The signal to be analyzed is decomposed in approximation an in detail while using two filter $\mathrm{h}$ and $\mathrm{g}$. The $\mathrm{h}$ filter is a low - pass filter with a band pass $[0, \Pi / 2]$, it generates the approximation signal "A".

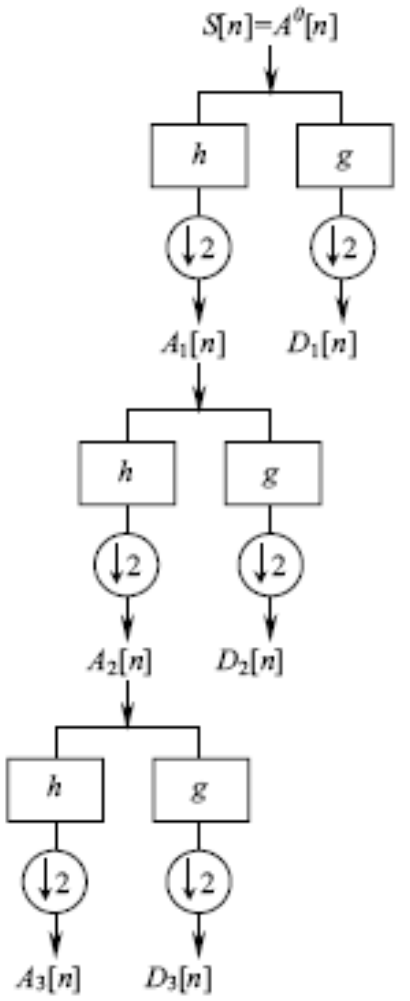

(a)
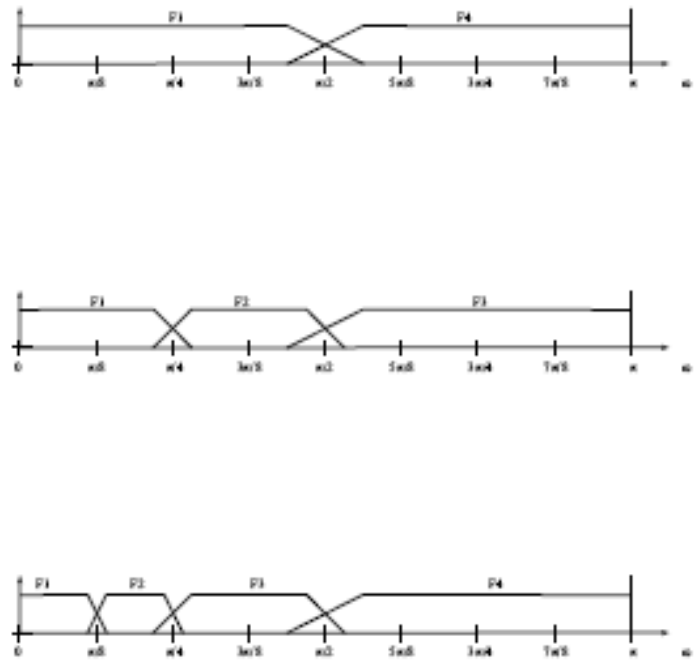

(b)

Fig. 2. a) Exemple of the discrete wavelet decompostion for three levels; b) Filter banks of the decomposition of each level 


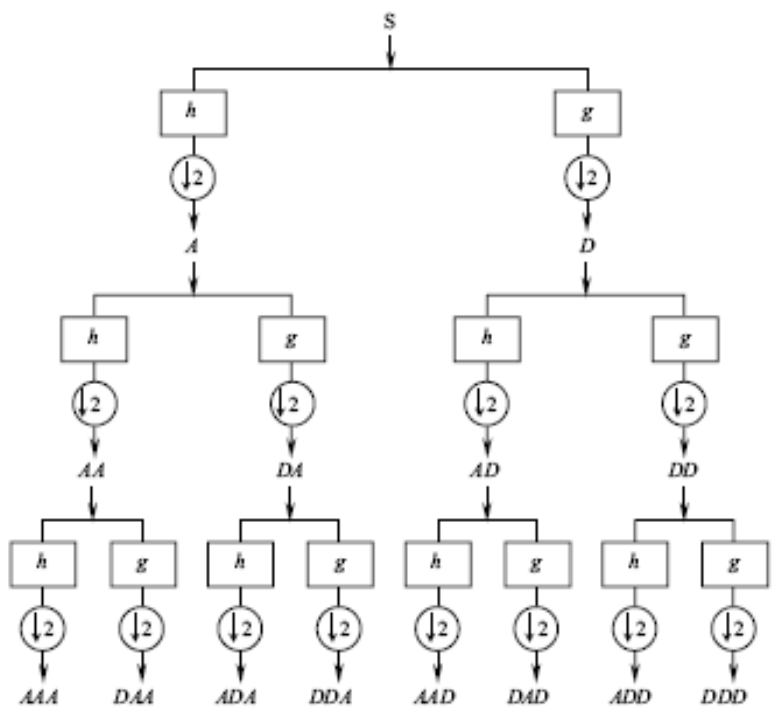

(a)
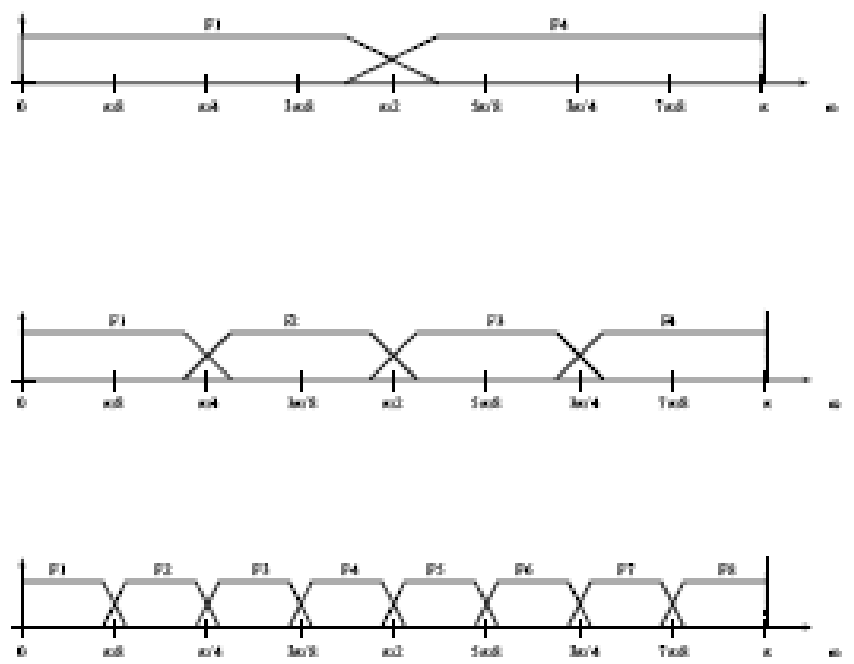

(b)

Fig. 3. a) Exemple of the packet wavelet decompostion for three levels; b) Filter banks of the decomposition of each level

\subsection{Wavelet Packet Transform (WPT)}

Wavelet packet analysis is an extension of the discrete wavelet transform (DWT) and it turns out that the DWT is only one of the much possible decomposition that could be performed 
on the signal. Instead of just decomposing the low frequency component, it is therefore possible to subdivide the whole time-frequency plane into different time-frequency pieces as can be seen from. The advantage of wavelet packet analysis is that it is possible to combine the different levels of decomposition in order to achieve the optimum time-frequency representation of the original [13].

To obtain a uniform filter bank we will use the wavelet packet analysis which is a generalization of the discrete wavelet transform analysis. The decomposition is also made of the details signal "D". Figure3 shows the wavelet packet decomposition on three levels as well as the filter banks associated for each uniform level.

\section{Results and discussion}

\subsection{Fast Fourier Transform application}

An FFT algorithm is first applied to the PCG signal given in Figure1. The frequency spectrum illustrated in Figure4a shows that the normal PCG signal has a frequency content varying from around $40 \mathrm{~Hz}$ up to $200 \mathrm{~Hz}$. The FFT can be applied to the first part of this signal to analyse the frequency content of $\mathrm{S} 1$ as shown in Figure $4 \mathrm{~b}$ and then to the second half to analyse the frequency content of S2 as shown in Figure4c. A 512 points FFT is applied to S1 and S2. At this stage the sound S1 or S2 cannot be separated.

In fact; the application of the FFT on heart sounds S1 and S2 after their separation or identification show that the basic frequency components are obviously detected by the Fourier transform .

The spectrum of S1 has reasonable values in the range $10-180 \mathrm{~Hz}$. The spectrum is distinctly resolved in time into two majors components (M1 and T1) while the most of the energy of these sounds appears to be concentrated.

The two components A2 (due to the closure of the aortic valve) and P2 due to the closure of the pulmonary valve) of the second sound S2 are obvious in Figure4c. The spectrum of the sound S2 has reasonable values in the range $50-250 \mathrm{~Hz}$. The spectrum for this sound is distinctly resolved in time into two majors components (A2 and P2) as shown in Figure4c. However the FFT analysis of S2 cannot tell neither which of A2 and P2 precedes the other, nor the value of the time delay known as the "split" which separate them. For a normal heart activity usually A2 precedes P2 and the value of the split is lower than 30ms. This time delay between A2 and P2 is very important to detect some pathological cases. The sound S2 seem to have higher frequency content than that of $\mathrm{S} 1$ as shown in Figure4b and Figure4c.

The FFT is applied also to analyse fourth PCG signals one normal and three different marked pathological cases (the aortic-insufficiency, the aortic-stenosis and the mitralstenosis). These are illustrated in Figure5 along with the normal PCG signal. The basic frequency content is obviously different from that of the normal PCG signal. It is clearly shown that there is great loss of frequency component in each of the pathological case with respect to normal case. In addition except the aortic-insufficiency case where we note the apparition of frequency component higher than $200 \mathrm{~Hz}$, the other cases (mitral-stenosis and aortic-stenosis) present a frequency spectrum limited to $200 \mathrm{~Hz}$.

The aortic-insufficiency and the aortic-stenosis are two pathological cases resulting from a severe organic attack, which generally involves a disappearance of the aortic component A2 of the sound S2. This shown in their corresponding PCG frequency responses illustrated in Figure5, where we notice a lack in frequency contents in the range under $100 \mathrm{~Hz}$ compared to the normal case, where there is much more frequency component in this range. On the 
other hand the mitral-stenosi is rather a severe attack of the mitral valves thus involving a presystolic reinforcement as well as a bursting of the sound S1.
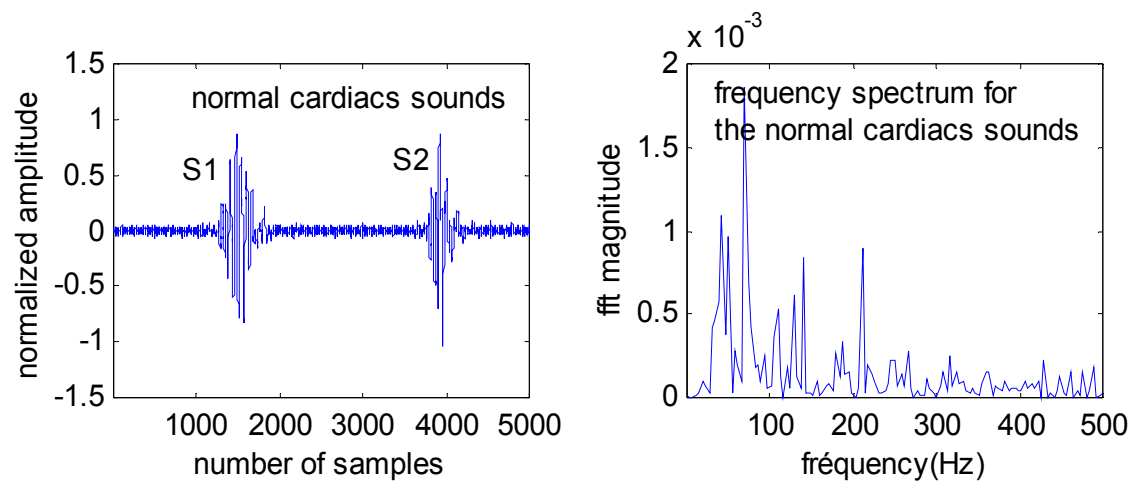

a)
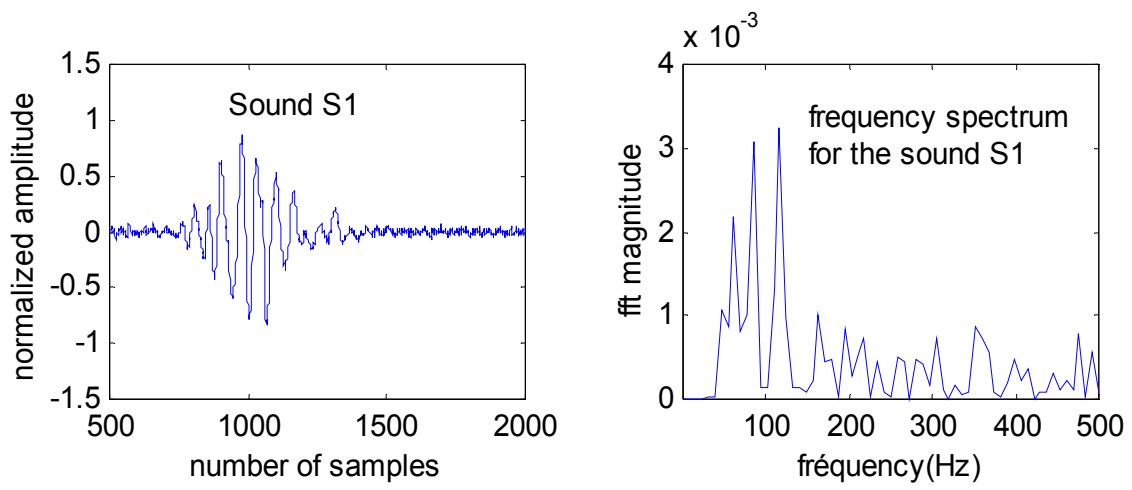

b)
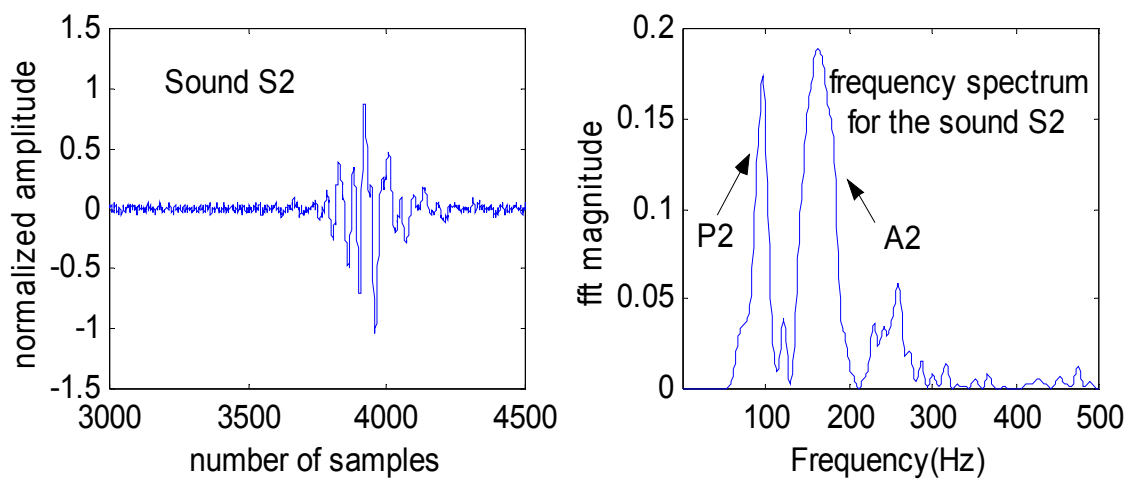

c)

Fig. 4. Frequency spectrum for the normal cardiac sounds and the sounds S1 and S2 

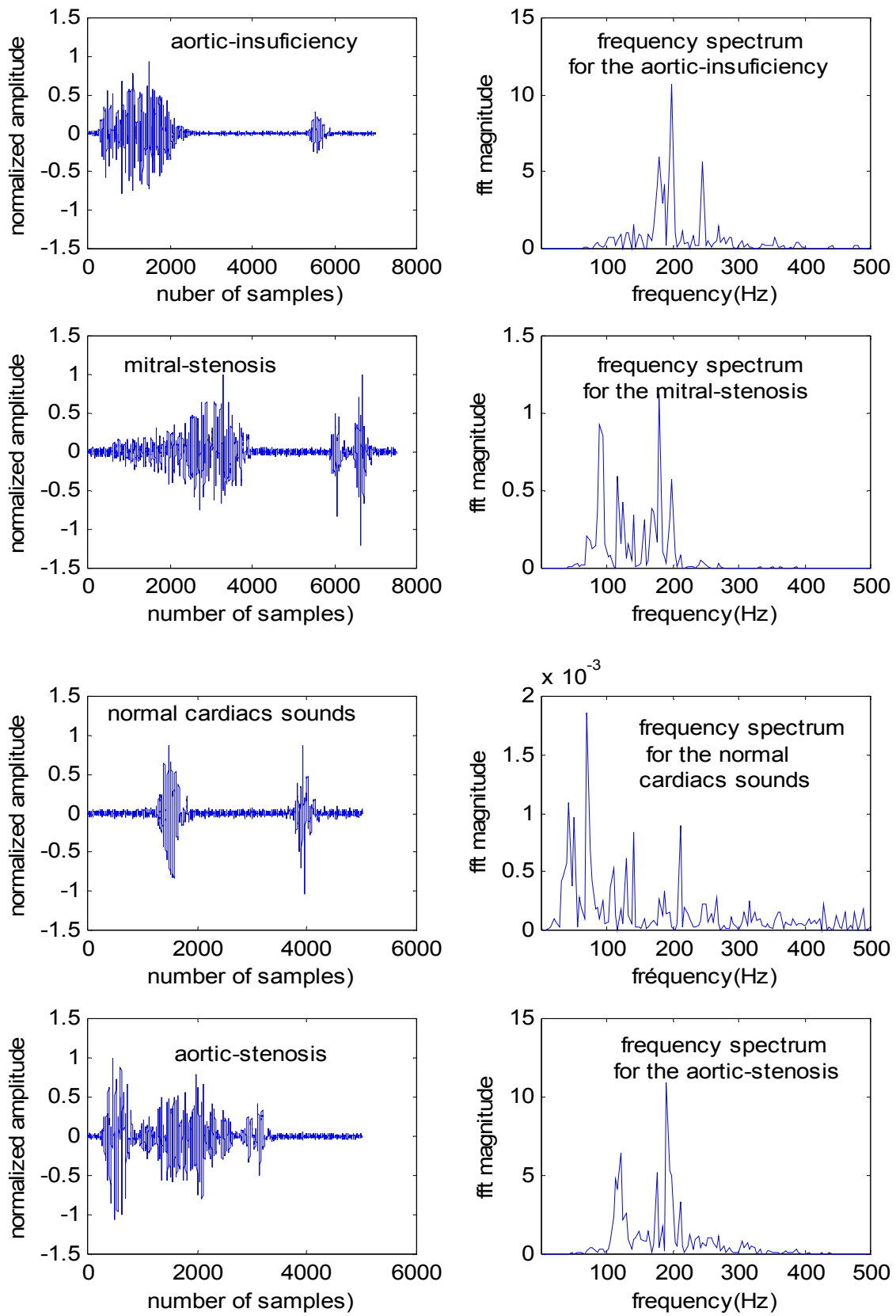

Fig. 5. Normal and abnormals cardiacs sounds and their frequency spectrum respectively 
As the frequency extent of the sound S1 is less important than that of the sound S2, the spectral response of the PCG signal related to this pathological case is not much affected comparad to that of the normal case as was the case in the aortic -insufficiency and aorticstenosis

In conclusion, and by applying the spectral analysis to different PCG signals, we can affirm which of the sounds S1 or S2 is directly concerned by the pathology, and more precisely which component of these sounds is affected.

With regard to normal PCG the basic frequency components are obviously detected by the FFT but not the time delay between these components. In fact as it was shown for example in Figure4c, the components A2 and P2 of the second sound S2 are obvious. However the FFT analysis of S2 cannot tell what is the value of the time delay between A2 and P2. It is thus essential to look for a transform which will describe a kind of "time-varying" spectrum.

\subsection{Short-time Fourier Transform application}

The normal phonocardiogram signal (Figure 6a) and the coarctation of the aorta (Figure 6a) are analyzed in this section. The coarctation of the aorta has been deliberately chosen here to evaluate the performance of the STFT analysis for it is very similar to the normal case. Figures 6a illustrate thus such a signal where we can notice that the temporal representation is almost similar to that of normal case given in Figure 6a.

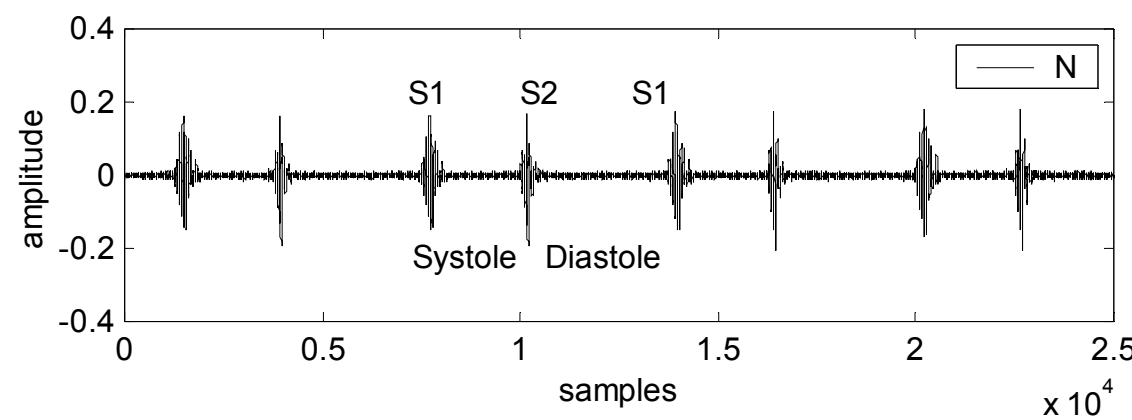

a)

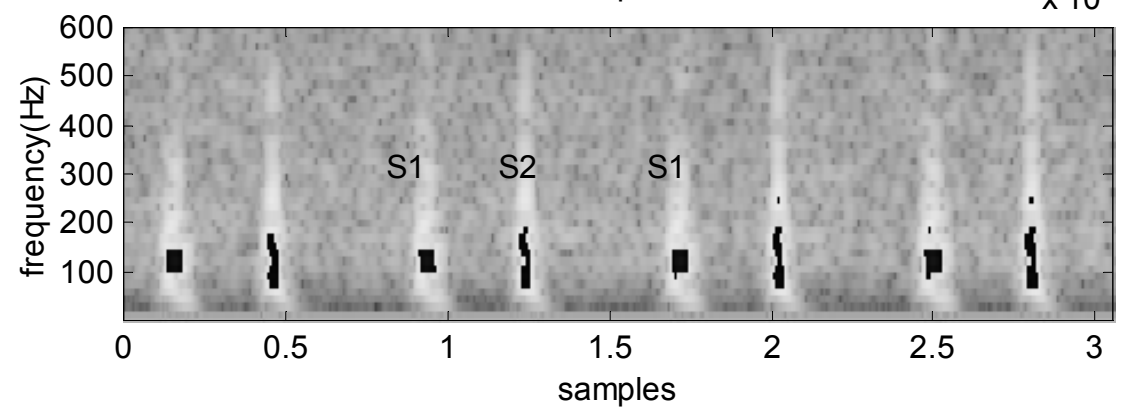

b)

Fig. 6. The STFT application of the normal PCG (N); a) the normal PCG signal (N); b) the spectrogram of the normal signal $(\mathrm{N})$

Figure $6 \mathrm{~b}$ and Figure $7 \mathrm{~b}$ provide respectively the STFT application of the normal and the CA case. From these Figures we can see the difference of the time-frequency features between 
them. For the two cases ( $\mathrm{N}$ and $\mathrm{CA}$ ), the second sound (S2) is shown to have higher frequency content than of the first sound (S1) $[14,15]$. This expected since the amount of blood present in the cardiac chambers is smaller [16].

We consider here two examples of the phonocardiogram signals with murmur: the pulmonary stenosis (PS :Figure 8a) with a systolic murmur and the aortic regurgitation (AR : Figure 9a) with a diastolic murmur.

Figure $8 \mathrm{~b}$ and Figure $9 \mathrm{~b}$ shows respectively the STFT application of the PS and AR signals. We can notice that the frequency extent of the diastolic murmur of the AR case is highly (About $600 \mathrm{~Hz}$ ) than the systolic murmur of the PS case (about $400 \mathrm{~Hz}$ ).

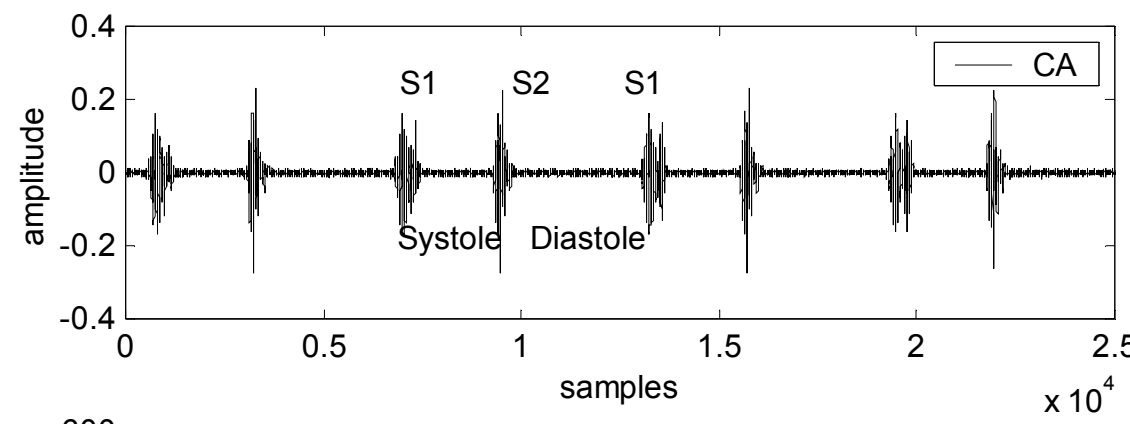

a)

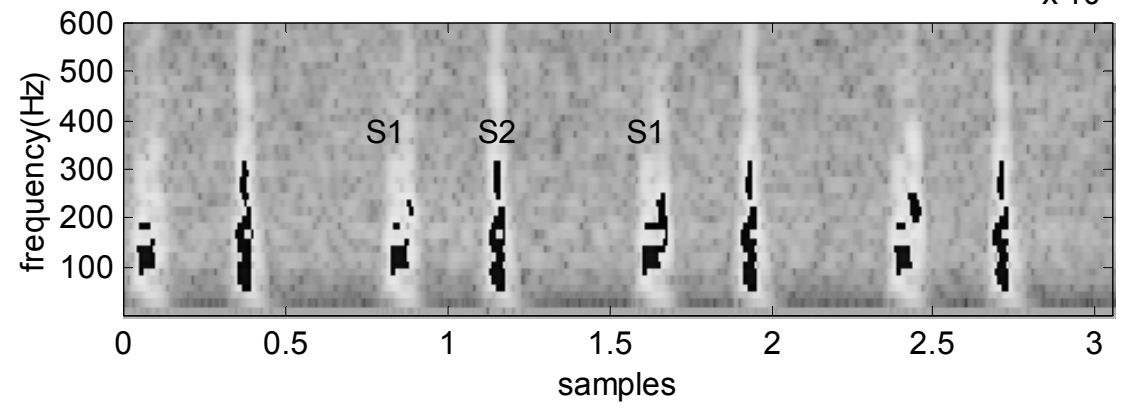

b)

Fig. 7. The STFT application of the Coarctation of the aorta (CA); a) the Coarctation of the aorta signal (CA); b) the spectrogram of the signal CA

In this section we presents the experimental results of the short-time frequency transform application of the three followings groups of the PCG signals used.

- Group 1 : normal (N) or similar morphological signal (CA) ;

- Group 2 : opening snap (OS) and ejection click (EC);

- Group 3: PCG signal with width murmur (PS and AR).

Figure 10 provides a better representation of the results obtained concerning the frequency contents of the sounds and murmurs analysed. If under the normal conditions $(\mathrm{N})$ or in the presence of similar signals (CA) the frequency content of the sound S2 is more significant than that of the sound $\mathrm{S} 1$.

We noted that the light murmurs (OS, EC.) can influence the time-frequency content of the principal sounds S1 and S2 and have a frequency extent more significant than them. 

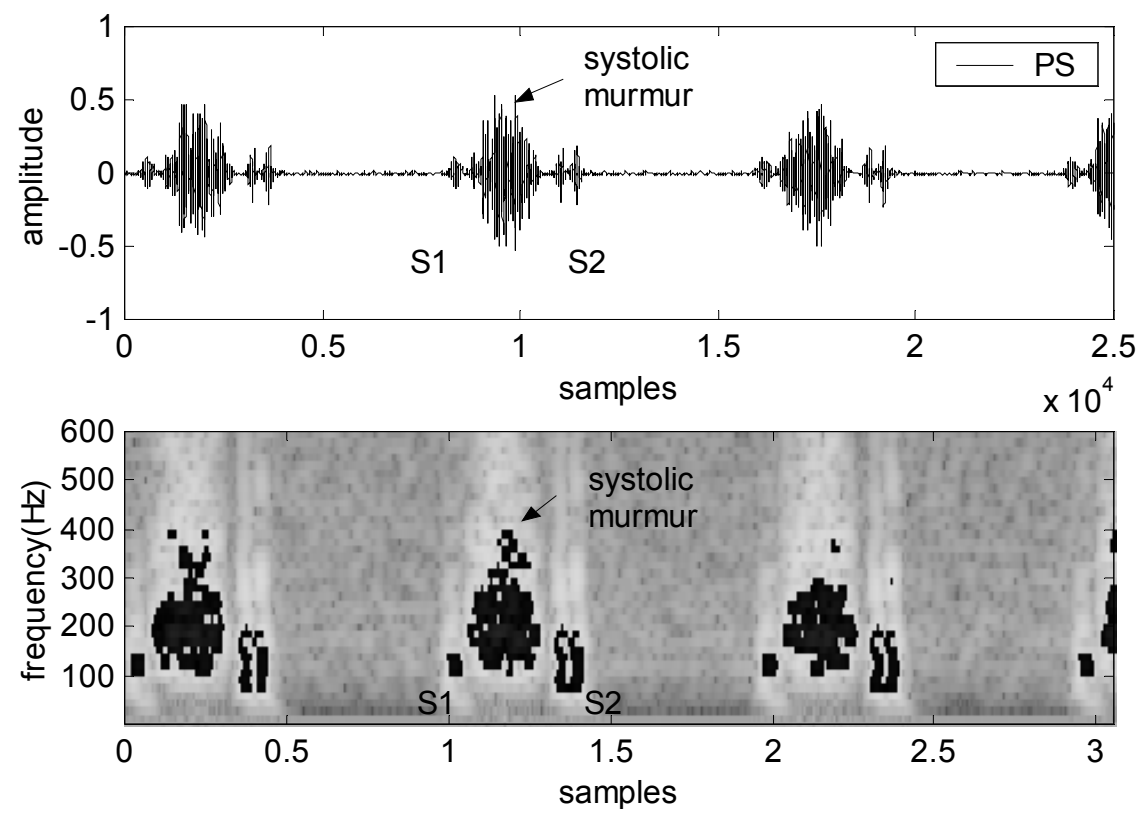

Fig. 8. The STFT application of the pulmonary stenosis; a) The pulmonary stenosis signal (PS); b) The spectrogram of the signal PS
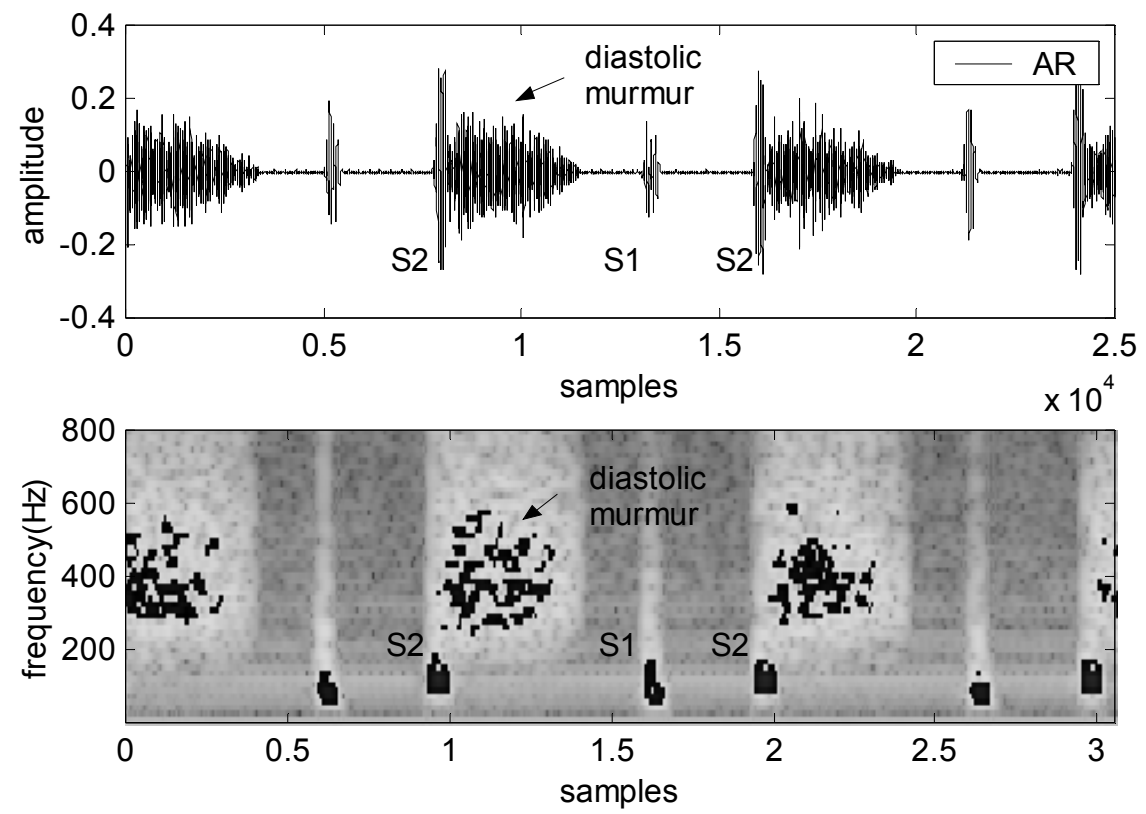

Fig. 9. The STFT application of the aortic of the regurgitation; a)The aortic of the regurgitation signal (AR); b)The spectrogram of the signal AR 
Finally in fact the width murmurs (PS and AR cases) present a frequency extent very significant. Discrimination between the systolic and diastolic murmurs can be made starting from this frequency extent, diastolic murmurs thus having a frequency extent more significant than the systolic murmurs. In more these murmurs seem not too much not to affect the time-frequency content of the sounds S1 and S2

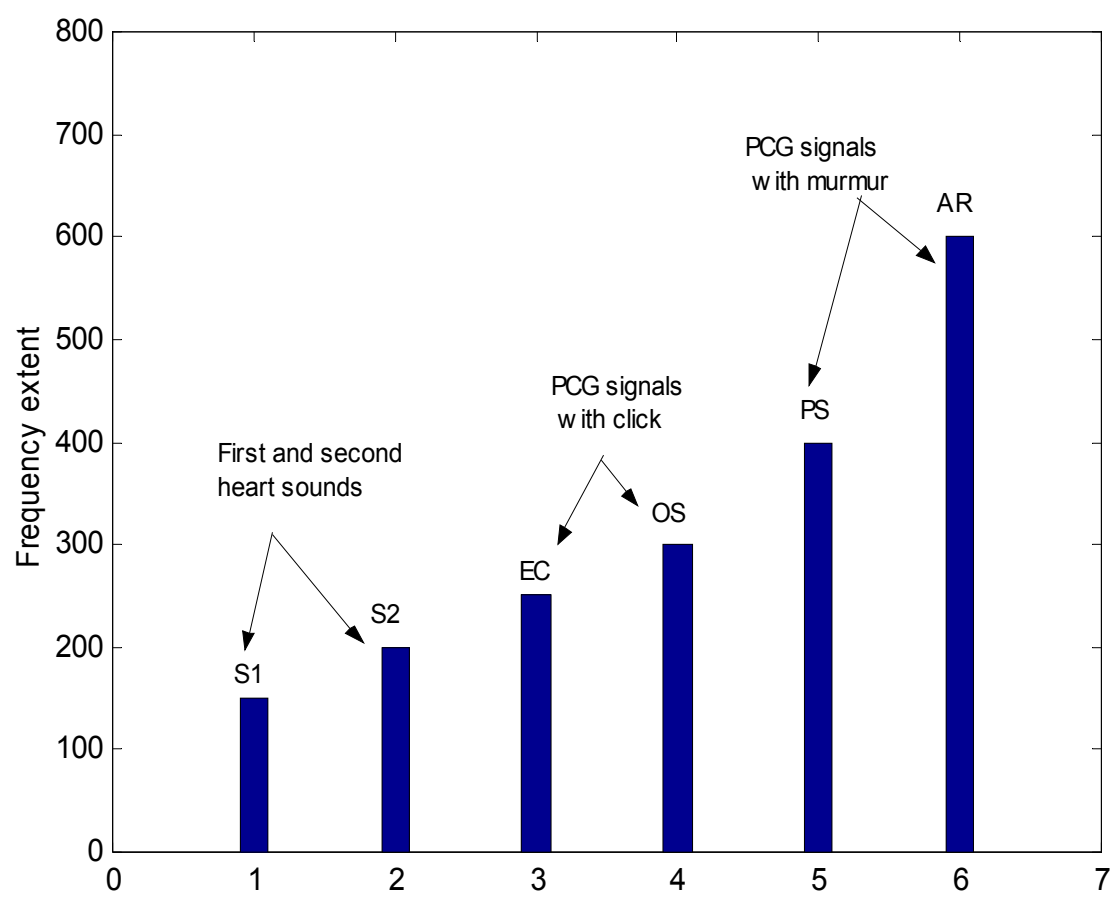

Fig. 10. Frequency extent of the three groups of the PCG signals

\subsection{Wigner Distribution application}

Figure11 shows the WD application of the cardiac sound of the normal case (11a), the coarctation of the aorta (11b) and the innocent murmur (11c). One can notice here that the two principal components (A2 and P2) start to appear in the presence cross-terms. The WD results may be improved by increasing the sampling rate of original signal,, but it still suffers from the cross-terms problem because of the nonlinearity of the WD.

However the Wigner distribution (WD) have shown good performances in the analysis of non-stationary signals. This comes from the ability of the WD to separate signals in both time and frequency directions. One advantage of the WD over the STFT is that it does not suffer from the time-frequency trade-off problem. On the other hand, the WD has a disadvantage since it shows cross-terms in its response. These cross-terms are due to the nonlinear behaviour of the WD, and bear no physical meaning. One way to remove these cross-terms is by smoothing the time-frequency plane, but this will be at the expense of decreased resolution in both time and frequency 


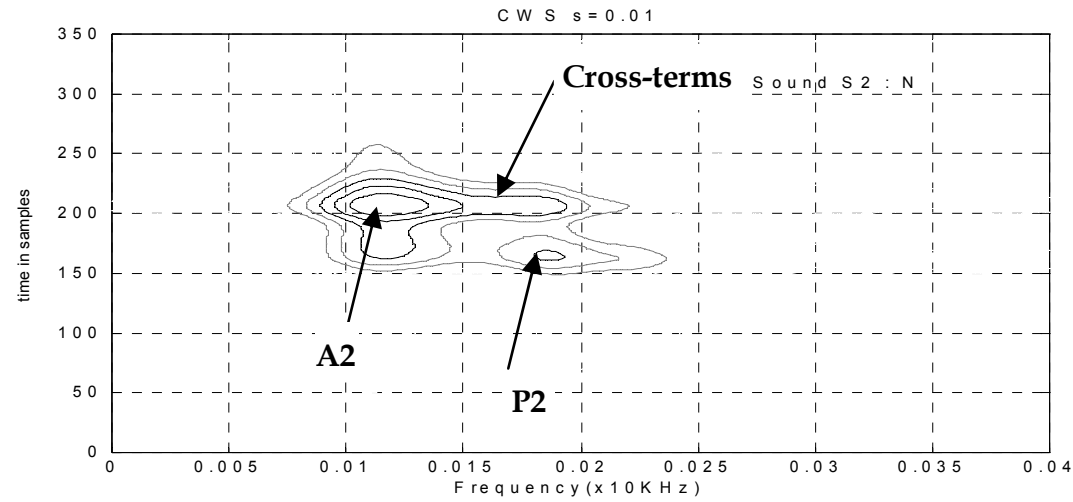

a)

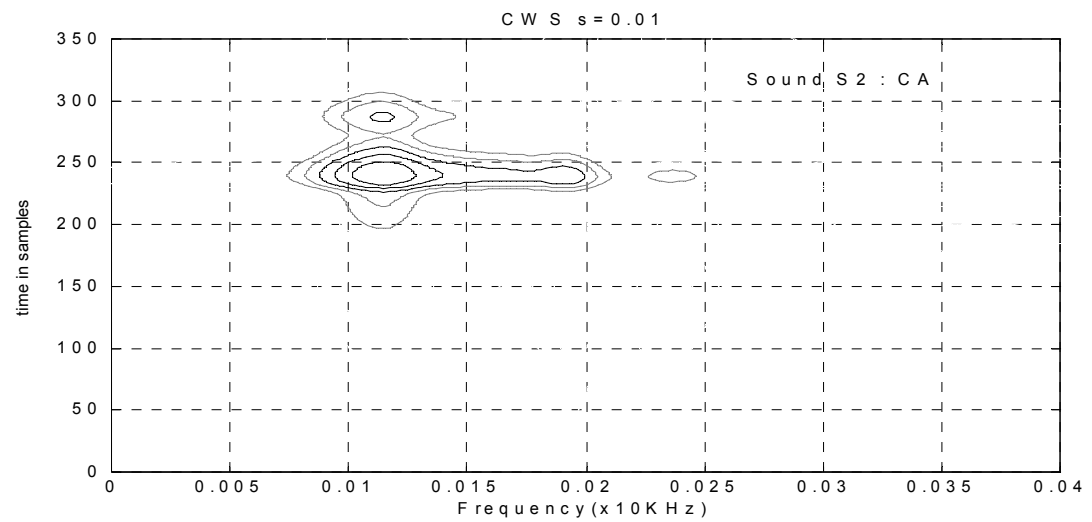

b)

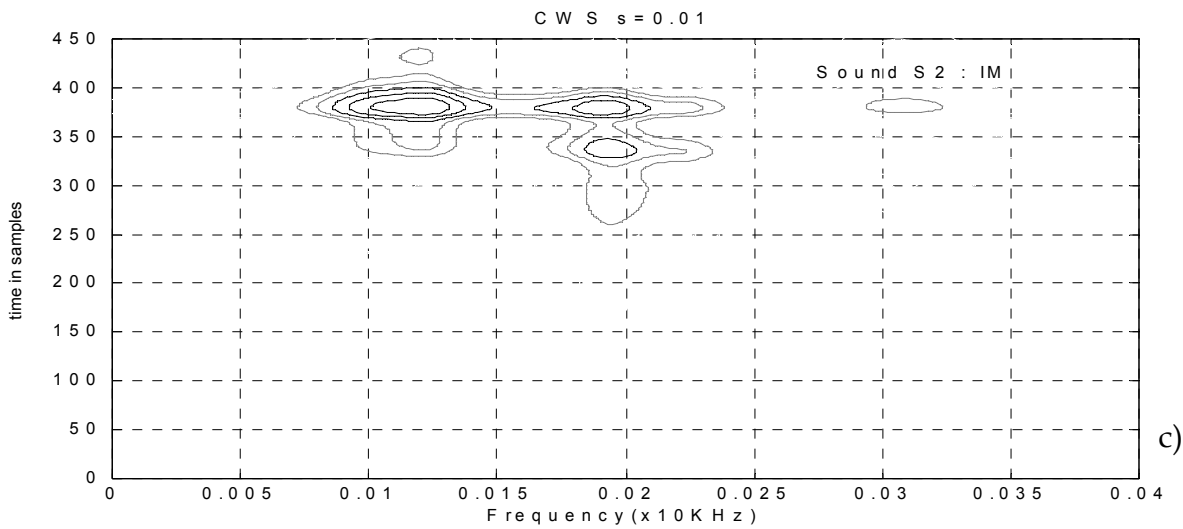

Fig. 11. The wigner distribution of the second cardiac sound S2 : a) the normal case , b) the coarctation of the aorta case (CA), c) the innocent murmur case (IM) 


\subsection{Continuous Wavelet Transform application}

An algorithm under MATLAB environnement of the Continuous Wavelet Transform is developed then applied to analyse the different PCG signals. First the normal PCG is studied as illustrated by Figure12.a. The two heart sounds are clearly shown in dark color. They are spaced with 2500 samples corresponding to 0.312 secondes. The continuous wavelet transforms of S1 and S2 are also displayed separately in Figure12.b and Figure12.c respectively. The sound S2 is shown to have higher frequency content than that of the S1. This is expected since the amount of blood present in the cardiac chambers is smaller [1],[16]. On Figure $12 \mathrm{~b}$ we can see that $\mathrm{S} 1$ is clearly resolved in two major component (M1 and T1). On the figure 10c the sound S2 is resolved also into two major's components (A2 and P2). The time delay between A2 and P2 can be easily measured with the use of the wavelet coefficients ( Figure12c). This delay is measured to be $13 \mathrm{~ms}$. It is smaller than the 30ms [17] as foreseen in the normal conditions of the PCG signal. Pathological conditions could cause this time difference to narrow or widen. Moreover, the order of occurrence of A2 and P2 may be reversed. The wavelet transform allows measurement and determination of this time difference, and thus allows a diagnosis process regarding this important parameter to be produced. Table I resumes the differences observed between the components A2 and P2.

\begin{tabular}{|c|c|c|c|c|}
\hline & $\begin{array}{c}\text { Localisation of the } \\
\text { Delay “d" } \\
(\mathrm{ms})\end{array}$ & $\begin{array}{c}\text { Minimal } \\
\text { frequency } \\
\text { (in scale) }\end{array}$ & $\begin{array}{c}\text { Maximal } \\
\text { frequency } \\
\text { (in scale) }\end{array}$ & $\begin{array}{c}\text { Frequency } \\
\text { Extent }\end{array}$ \\
\hline A2 & 13 & 19 & 124 & 105 \\
\hline P2 & 13 & 18 & 116 & 98 \\
\hline
\end{tabular}

Table I. Temporal and frequential measurements related to the componentsA2 and P2.

It can be concluded for the normal PCG that :

1. The component $\mathrm{A} 2$ precedes in time the component $\mathrm{P} 2$.

2. The component $\mathrm{A} 2$ have higher frequency content than P2.

3. The amplitude of $\mathrm{A} 2$ is more important than that of $\mathrm{P} 2$.

These parameters, particularly the frequency, make it possible to see a difference between A2 and P2.

Moreover the ability of the wavelet transform in heart disease diagnosis can be studied by applying the CWT algorithm on different marked cases. The result of this application are illustrated in Figure13b (aortic-insufficiency), Figure13c (aortic-stenosis) Figure13d (mitralstenosis). The coefficients of the CWT allow us to clearly discern the frequency range of each signal. It also shows the major components according to the temporary variation; the maximal amplitude is characterised by a darker color than those of the small amplitudes.

\subsection{Discrete Wavelet Transform application}

The multiresolution analysis based on the discrete wavelet transform (DWT) is a powerful tool in and filtering, separating and identification of the internal components and murmurs of the various analyzed signals (Figure14).

Figure15a shows the application of the discrete wavelet transform of one cycle of the normal PCG. Levels $\mathrm{d} 1$ and $\mathrm{d} 2$ represent the high frequency variations of the base line of PCG signal. Levels $\mathrm{d} 3$ and $\mathrm{d} 4$ emphasize clearly the side of high frequency content of the sound S2 compared to S1The component A2 (the most predominant in sound S2) appears better on levels $\mathrm{d} 5$ and $\mathrm{d} 6$. Level $\mathrm{d} 7$ represents the two principal components of the sound S1 (M1, T1). 

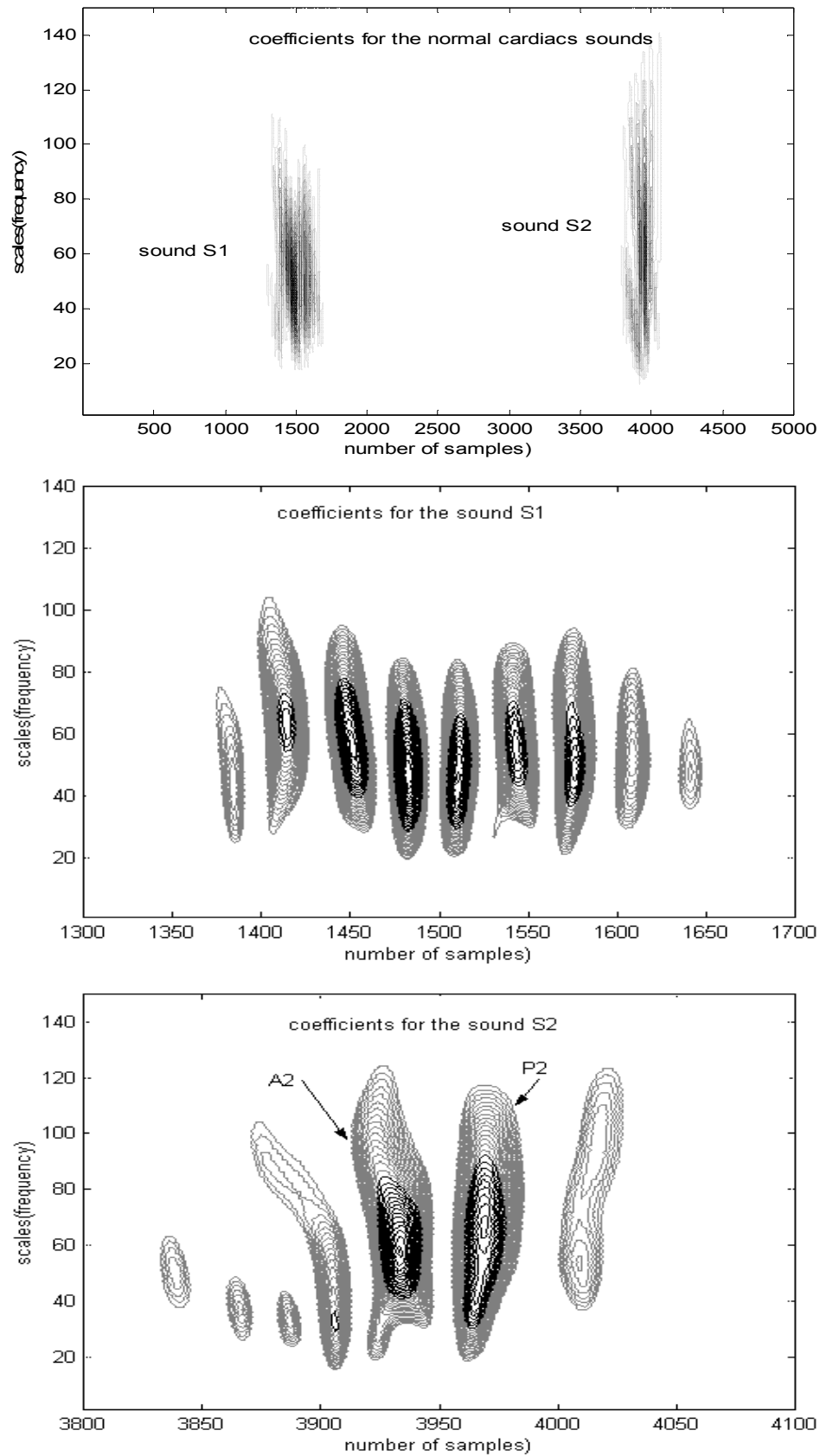

Fig. 12. Continuous wavelet transform analysis (CWT) for the normal cardiac sounds S1 and S2 


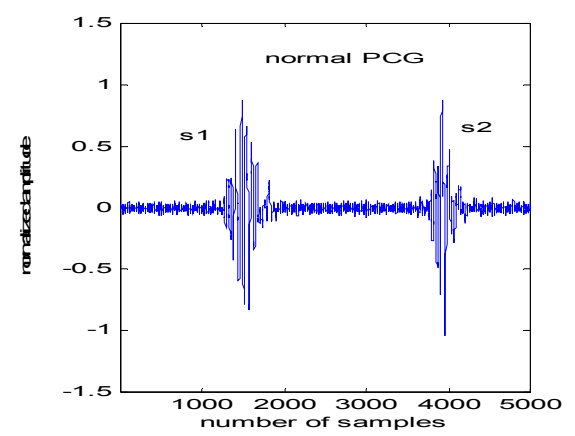

a)
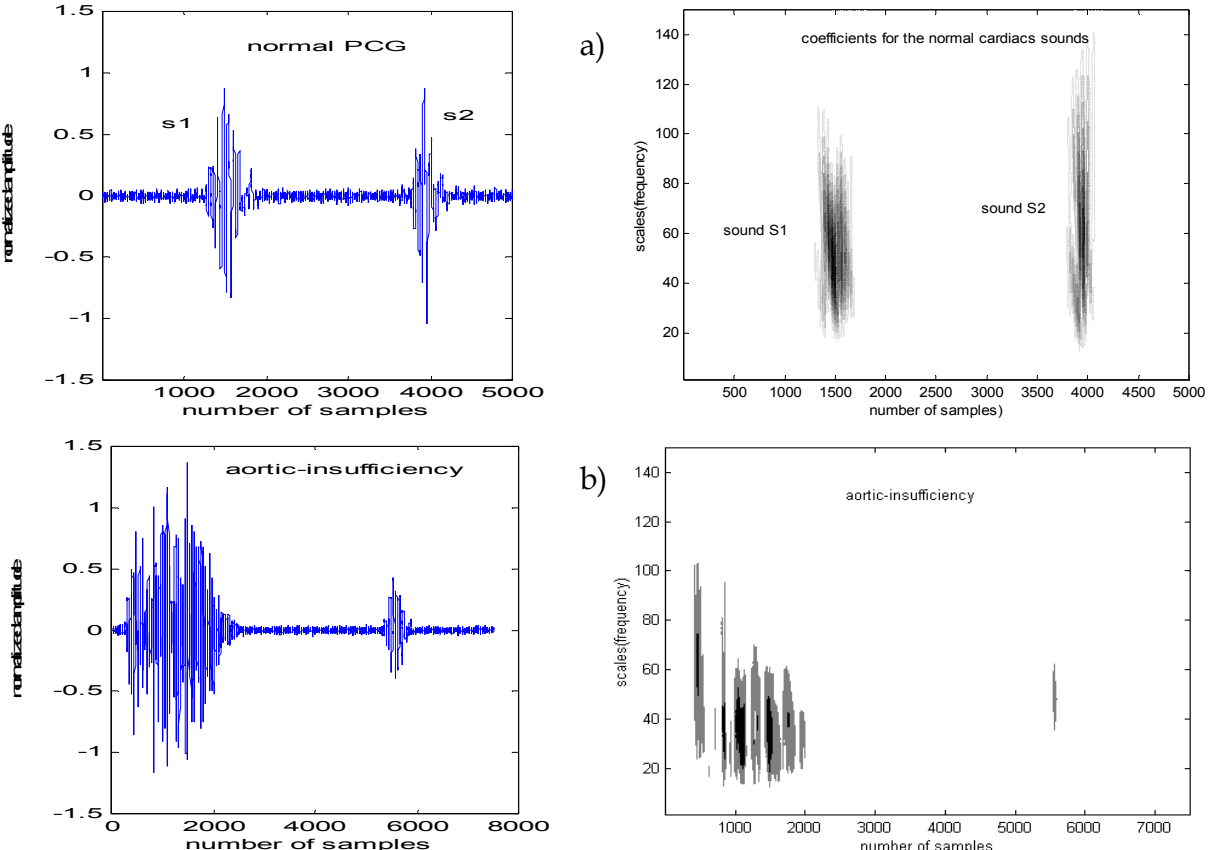

b)
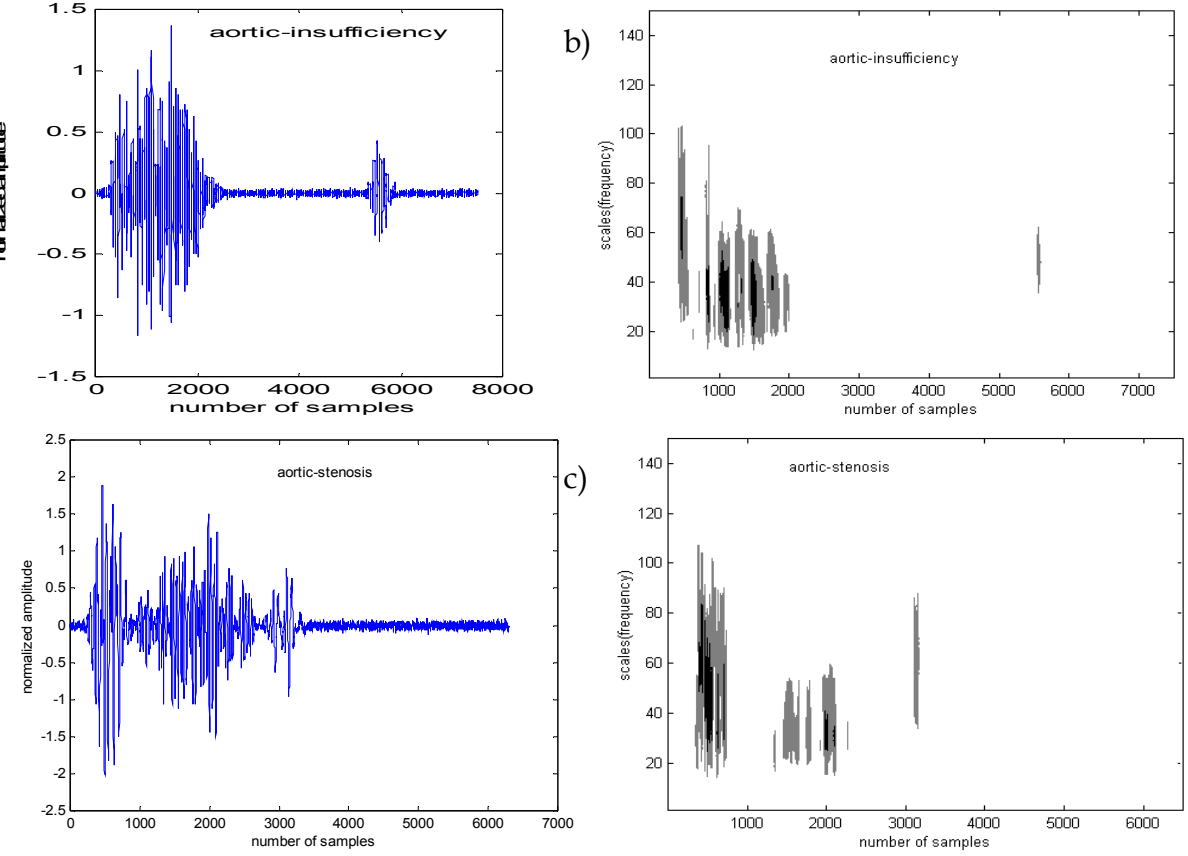

c)
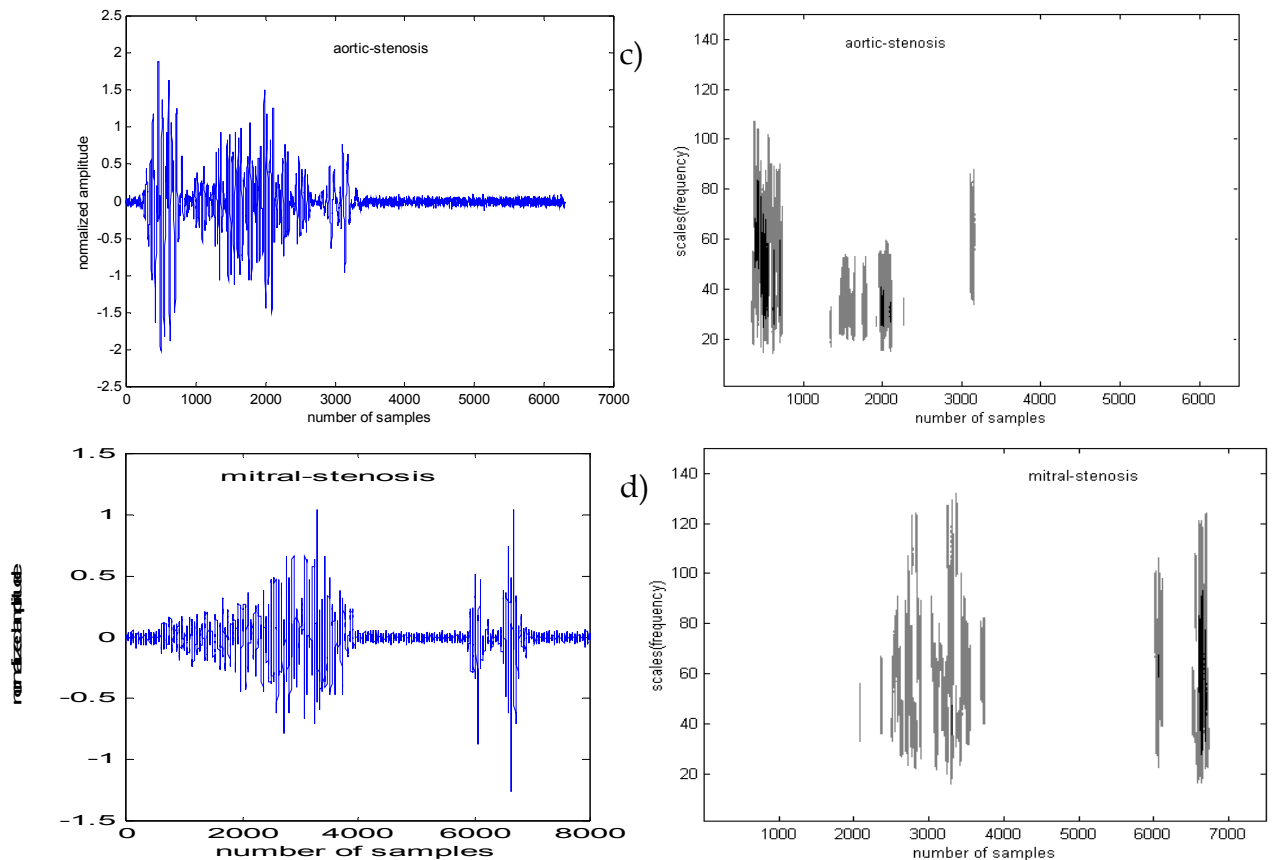

Fig. 13. Continuous wavelet analysis (CWT) for the normal PCG and abnormal PCG. 
The same analysis by using the discrete wavelet transform concerning the coarctation of the aorta and the mitral stenosis is also applied (Figures16b and Figure16c). Figure 13b, in level $\mathrm{d7}$, provide a well representation of the third component " $\mathrm{C}$ " added a the two majors components of the sound S2 (A2 and P2).

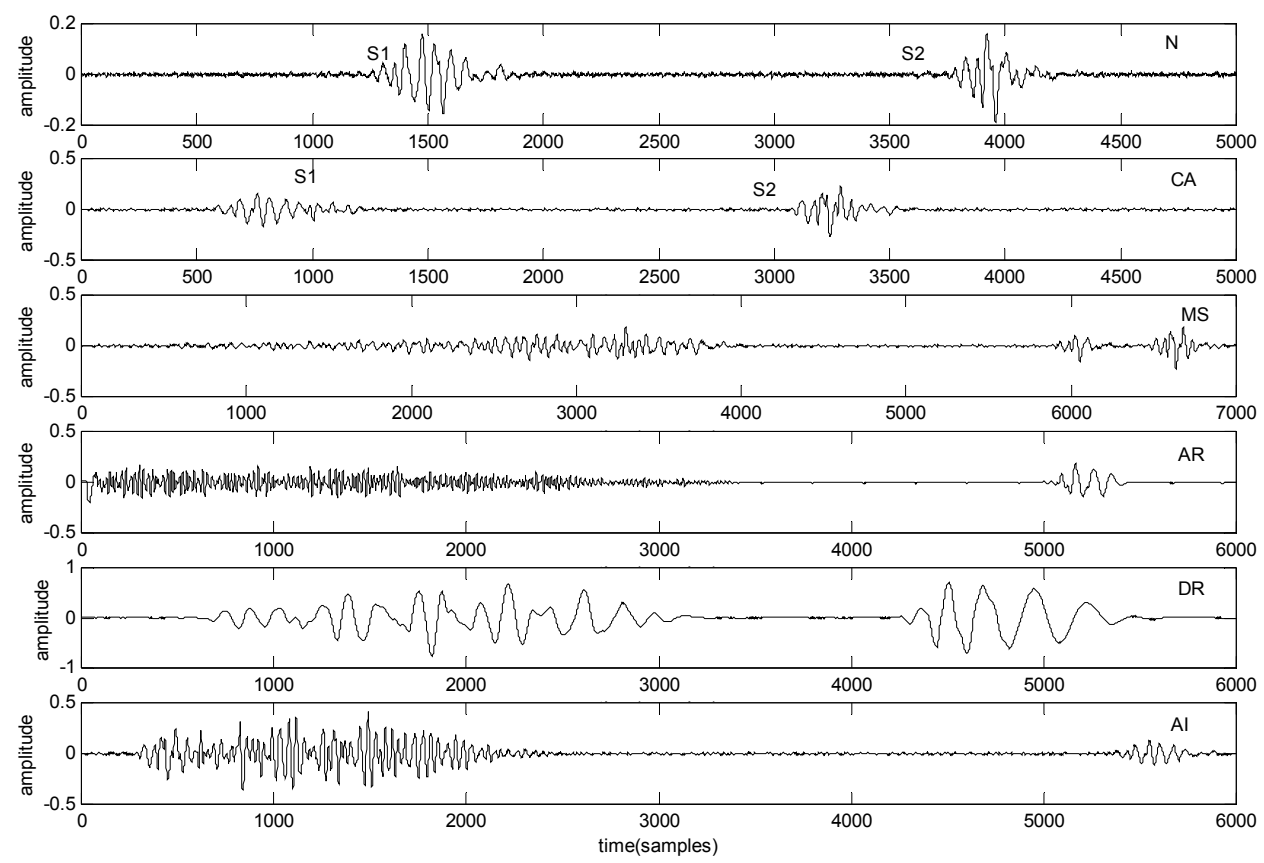

Fig. 14. PCG signals used a) normùal $(\mathrm{N}), \mathrm{b})$ the coarctation of the aorta $(\mathrm{CA})$; $\mathrm{c}$ ) the mitral stenosis (MS), d) the aortic regurgitation (AR), e) the diastolic ruble (DR) , f) the aortic insufficiency (AI)-

\subsection{Packet wavelet transform application}

The used of the wavelet packet transform is exactly the same as those developed in the discrete wavelet transform. The only difference is that wavelet packets offer a more complex and flexible analysis, because in wavelet packet analysis, the details as well as the approximations are split (Figure3). Single wavelet packet decomposition gives a lot of bases from which you can look for the best representation with respect to a design objective. The wavelet packet method is thus a generalization of wavelet decomposition that offers a richer range of possibilities for signal analysis. In wavelet analysis, a signal is split into an approximation and a detail. The approximation is then itself split into a second-level approximation and detail, and the process is repeated. 
The wavelet packet transform analysis in this paper give important features of the extent frequency of the heart sounds (S1 or S2) and cardiac murmur. These features can be help clinician in their diagnosis or recognizing pathological conditions concerning the recording PCG signals.

Figure 16 provide a time frequency (TF) representation of one cardiac cycle of the heart sounds concerning the normal PCG signal (Figure 16a), the coarctation of the aorta case (Figure 16b) and the mitral stenosis case (Figure 16c). These figures shown the frequency range of each component or murmur of the PCG signal studied. Thus we can observe the component "C" added of the two majors component for the sound S2 (A2 and P2). The same result have been find in the section 3.5 (Figure 14b.detail d7)

Figure 17 has the advantage of presenting at the same time the frequency extent of the various components of the cardiac sound like their frequency site one compared to the other. We can shown clearly that the Diastolic ruble (DR) case have high frequency content that the aortic insufficiency case (AI) or the aortic regurgitation case (AR).

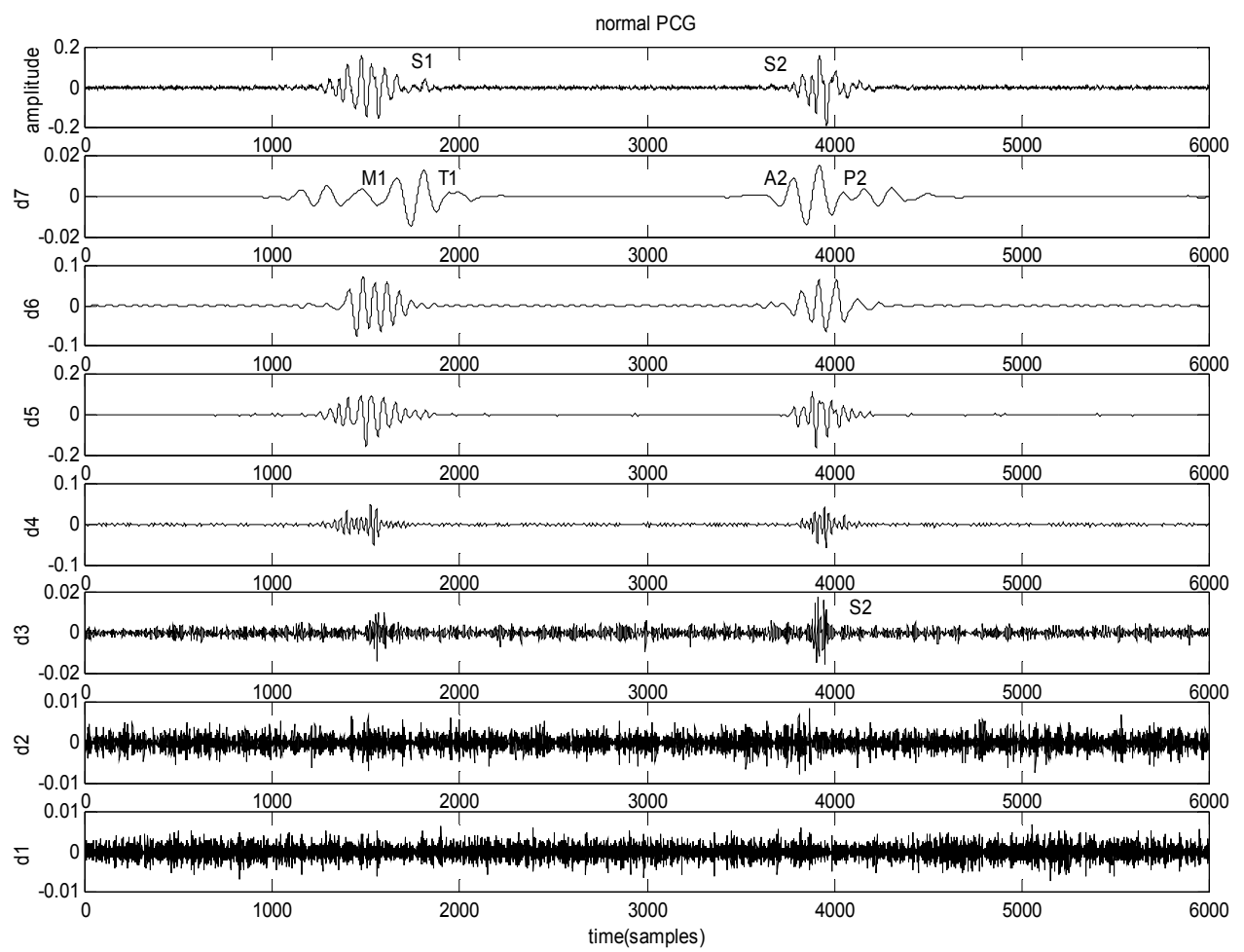

a) 


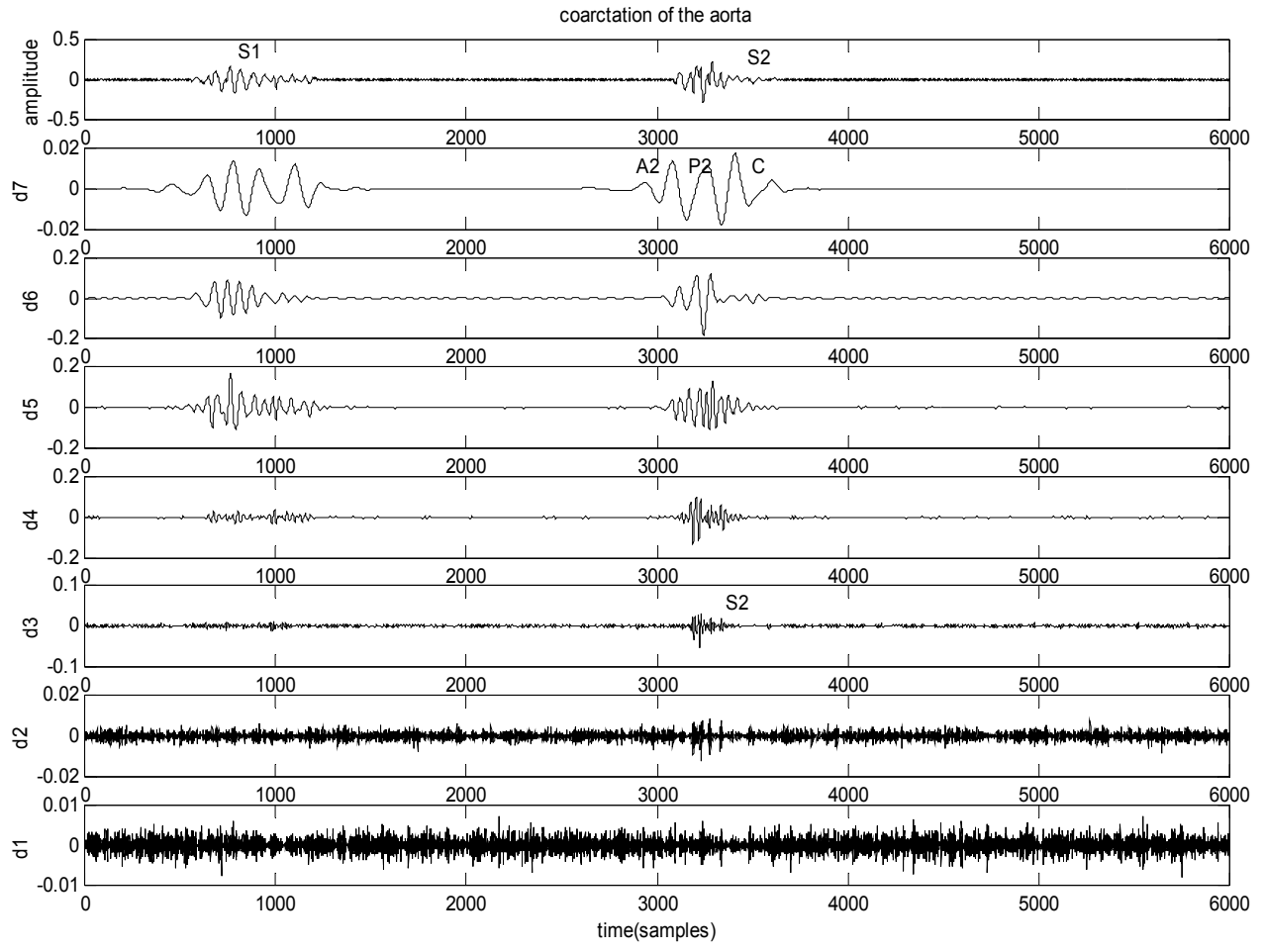

b) 


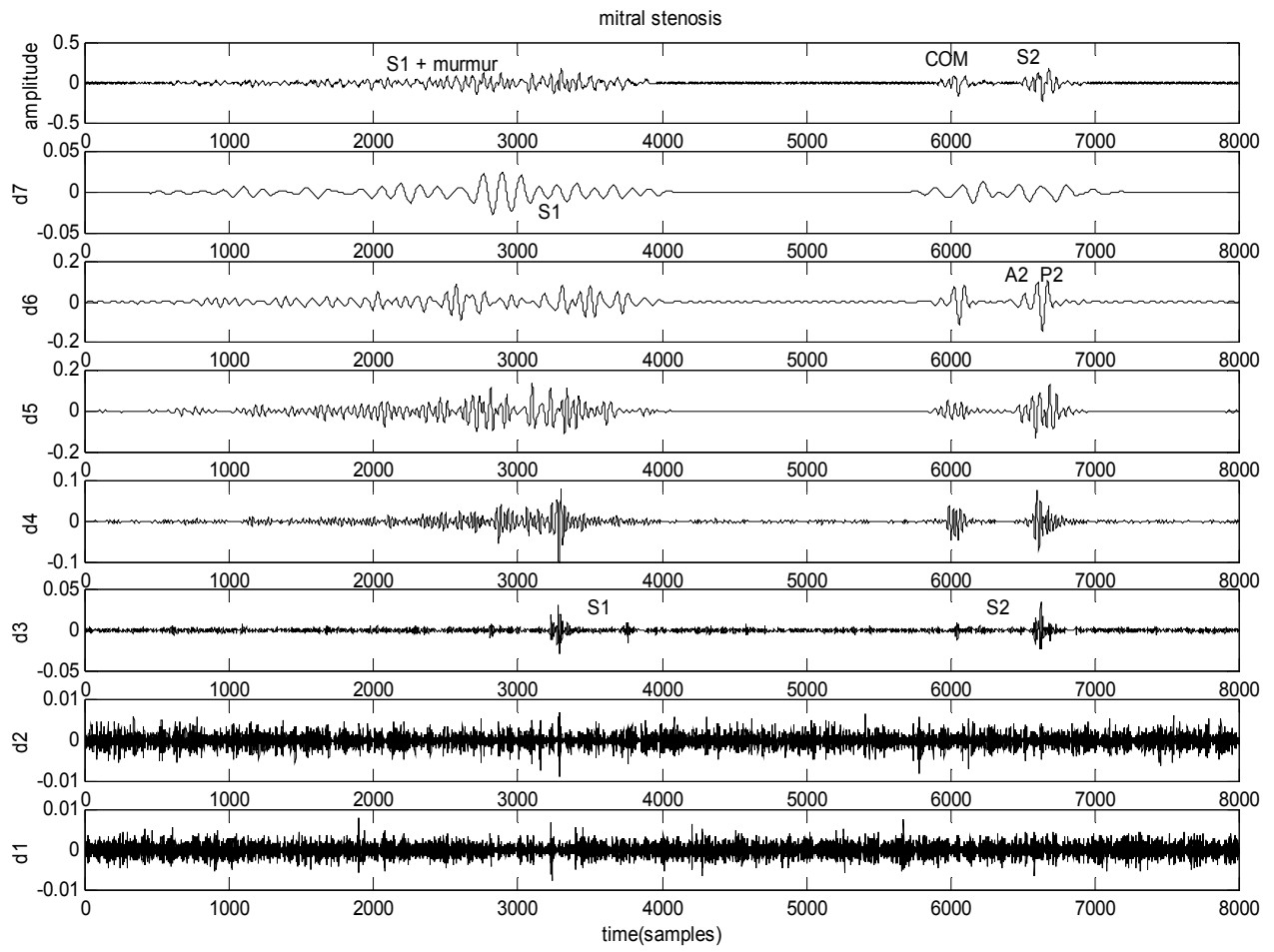

c)

Fig. 15. Discrete wavelet transform (DWT) analysis for: a) the normal PCG, b) the Coarctation of the aorta, c) the mitral stenosis 


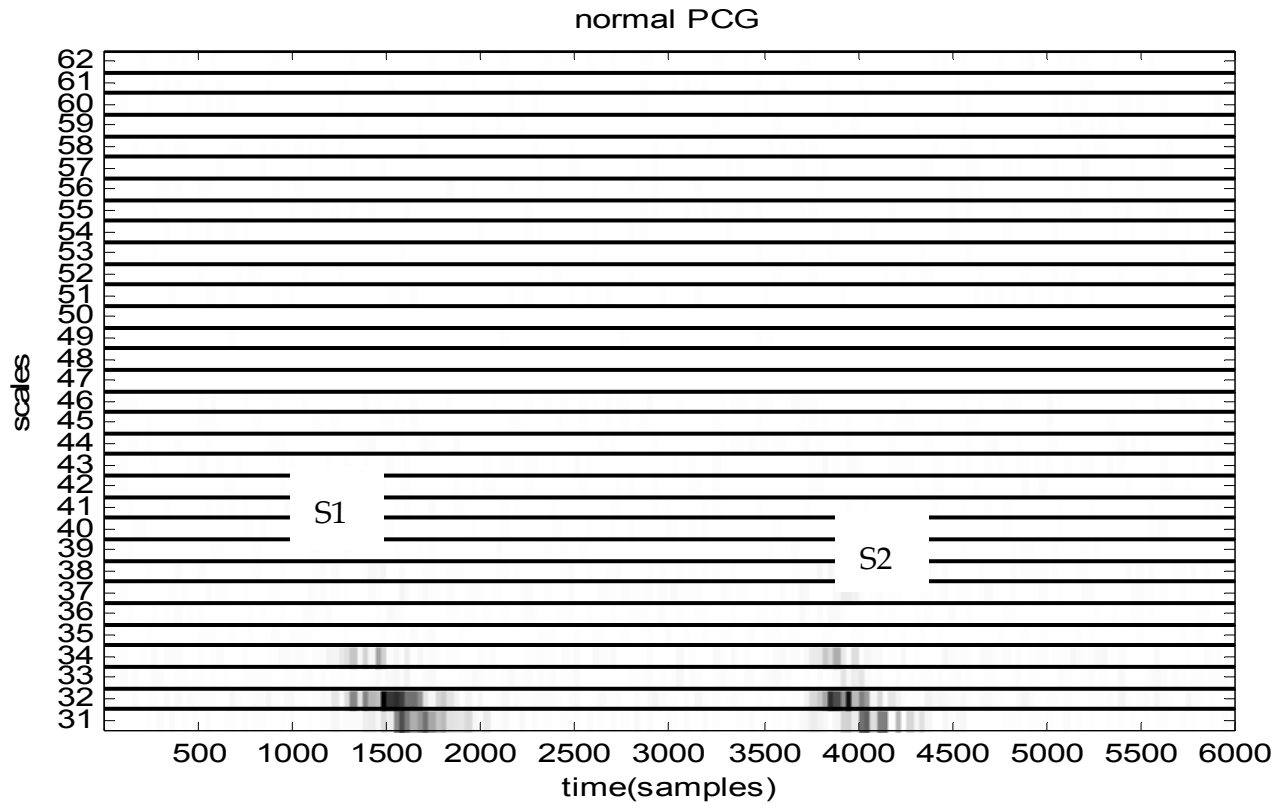

a)

coarctation of the aorta

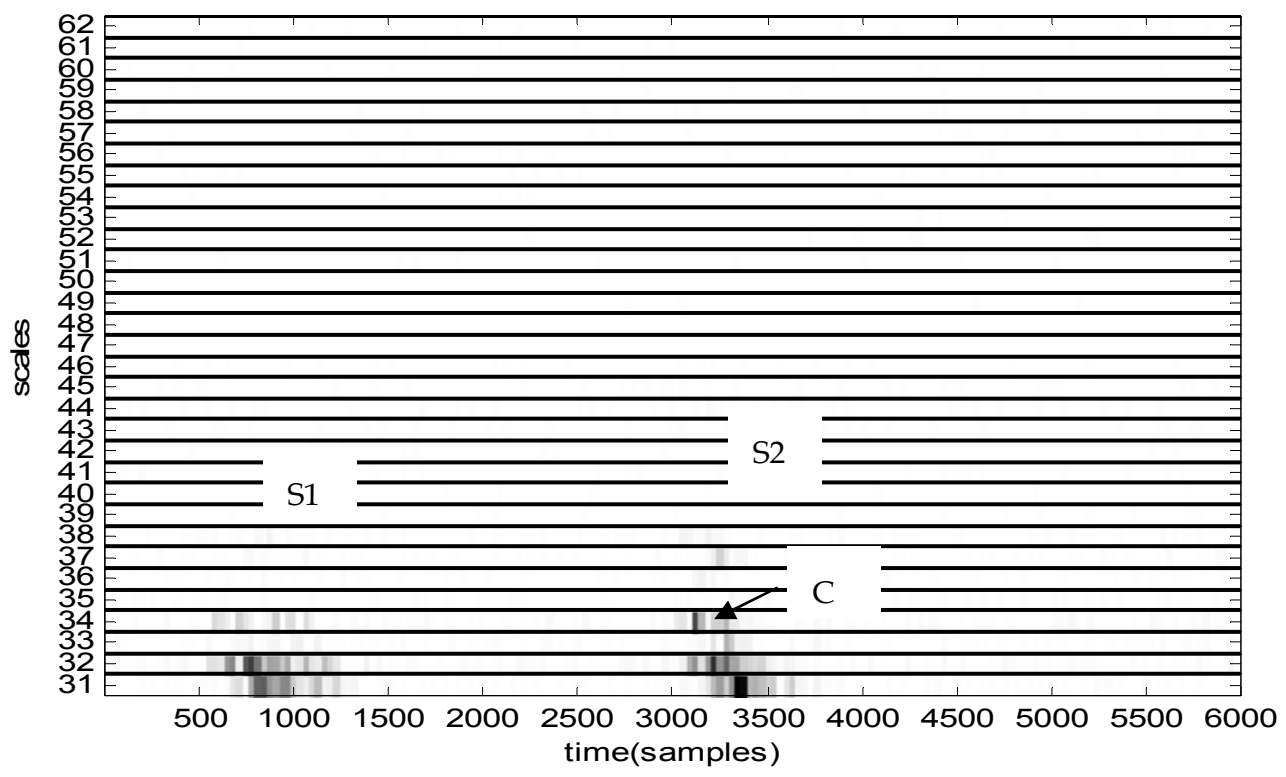

b) 


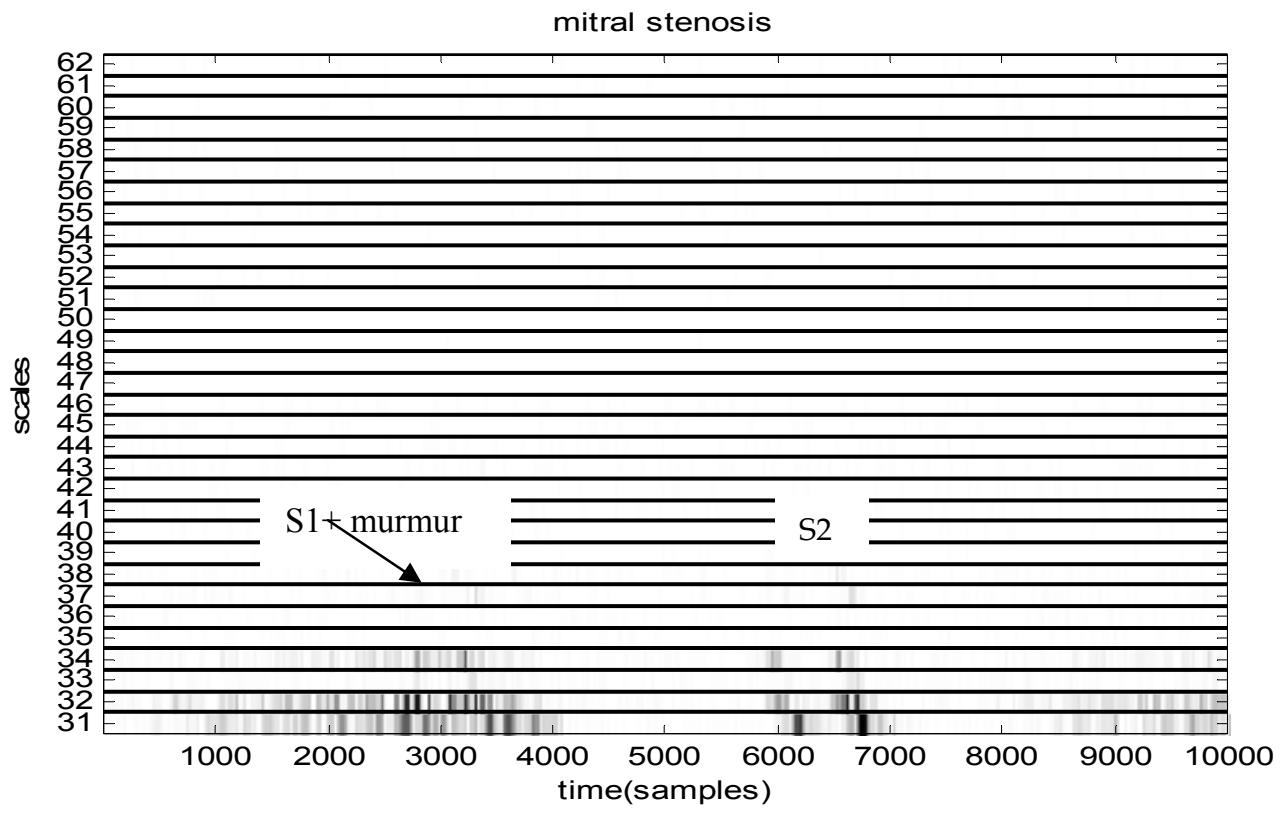

c)

Fig. 16. Wavelet packet transform (WPT) analysis for: a) the normal PCG, b) the coarctation of the aorta , c) the mitral stenosis

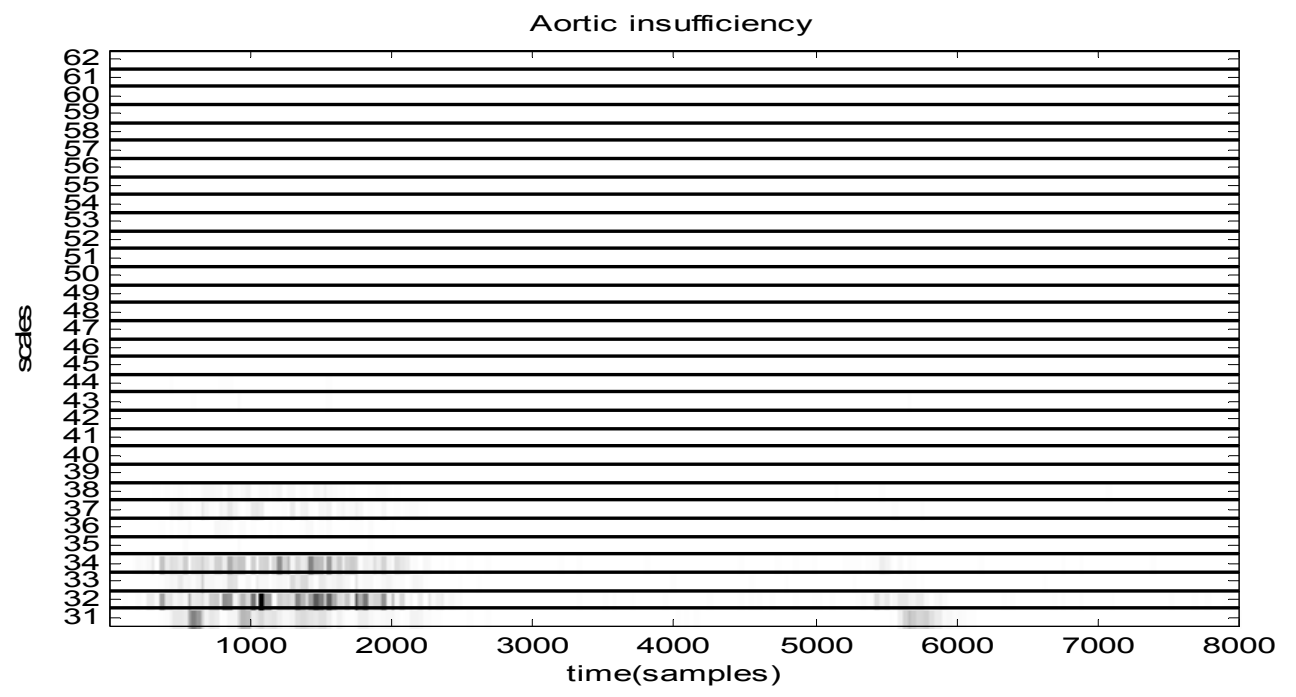

a) 


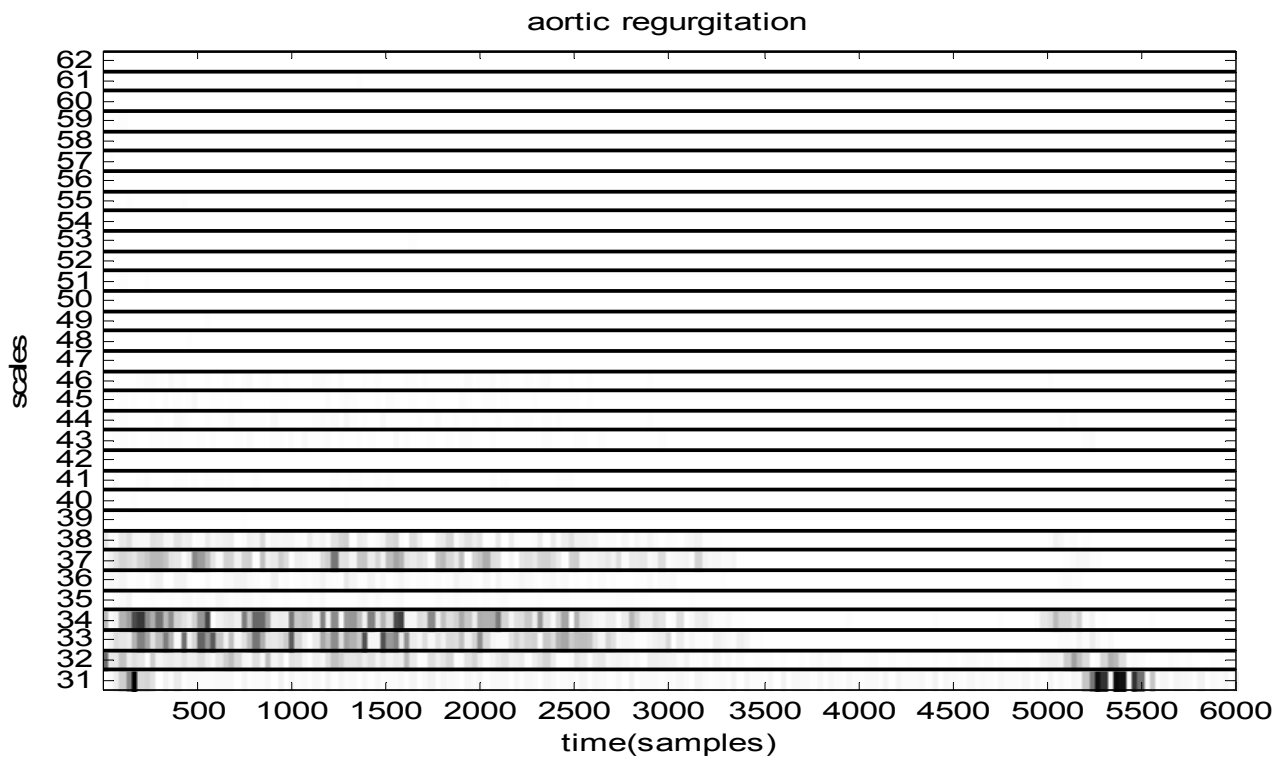

b)

\section{Diastolic Rumble}

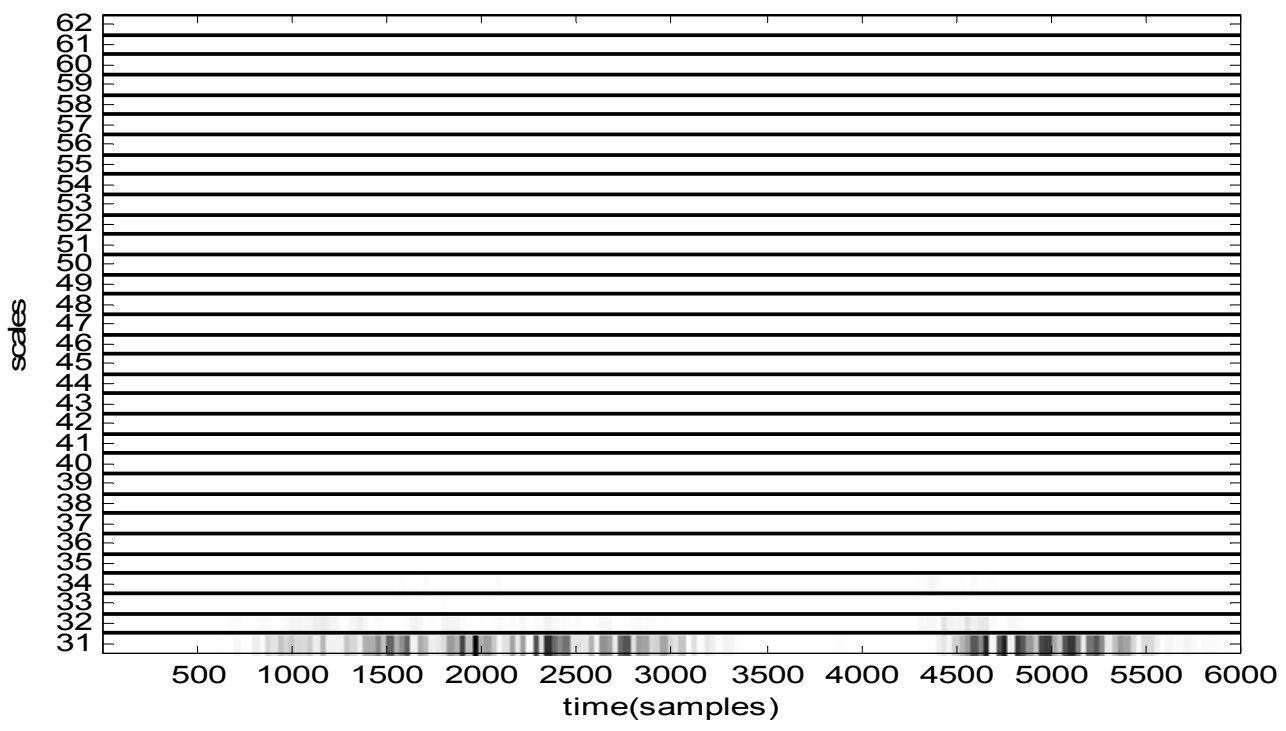

c)

Fig. 17. Wavelet packet Transform (WPT) analysis for: a) the aortic Insufficiency (Ai), b) the aortic regurgitation (AR), c) the Diastolic Rumble (DR) 


\section{Conclusion}

The cardiac (heartbeat sound) cycle of phonocardiogram (PCG) is characterized by transients and fast changes in frequency as time progresses. It was shown that basic frequency content of PCG signal can be easily provided using FFT technique. However, time duration and transient variation cannot be resolved; the CWT wavelet transform therefore i is a suitable technique to analyse such a signal. It was also shown that the coefficients of the continuous wavelet transform give a graphic representation that provides a quantitative analysis simultaneously in time and frequency. It is therefore very helpful in extracting clinically useful information.

The measurement of the time difference between the A2 and P2 components in the sound $\mathrm{S} 2$, the number of major components of the sounds S1 and S2 and the frequency range and duration for all these components and sounds can be accurately achieved for the CWT simultaneously as was clearly illustrated.

It is found that the wavelets transform is capable of detecting the four major components of the first sound S1 and the two components (the aortic valve component A2 and the pulmonary valve component P2) of the second sound S2 of a normal PCG signal. These components are not accurately detectable using the STFT or WD. However the standard FFT can display the frequencies of the components A2 and P2 but cannot display the time delay between them.

The application of the STFT in the analysis of the PCG signals made it possible to obtain appreciable information on the time-frequency content of the sounds S1, S2 and of the added murmurs (OS,EC or width murmurs).

If under the normal conditions $(\mathrm{N})$ or in the presence of similar signals (CA) the frequency content of the sound S2 is more significant than that of the sound S1. We noted that the light murmurs (OS, EC.) can influence the time-frequency content of the principal sounds S1 and $\mathrm{S} 2$ and have a frequency extent more significant than them.

Finally in fact the width murmurs (PS and AR cases) present a frequency extent very significant. Discrimination between the systolic and diastolic murmurs can be made starting from this frequency extent, diastolic murmurs thus having a frequency extent more significant than the systolic murmurs. In more these murmurs seem not too much not to affect the time-frequency content of the sounds S1 and S2.

The two version of analysis of the wavelet transform (DWT and PWT) make it possible to gather time-frequency information concerning the characteristics of the cardiac sounds.

It is shown that the FFFT, the STFT, the WD and the WT techniques provides more information of the PCG signals with murmurs that will help physicians to obtain qualitative and quantitative measurements of the time and the time-frequency PCG signal characteristics and consequently aid to diagnosis.

\section{References}

[1] Rangayyan,R.M and Lehner ,R.J (1988). A review.CRC Critical Reviews in Biomedical Engineering 15 (3), 211-236.

[2] Rangayyan,R.M and Lehner ,R.J (1988). Phonocardiogram signal analysis: a review. CRC Critical Reviews in Biomedical Engineering 15 (3), 211-236. 
[3] Feigen , L.P (1971). Physical characteristics of sound and hearing. American journal of Cardiology, 28 (2), 130-133 .

[4] Tuteur, F.B (1988). Wavelet Transforms in signal detection. IEEE ICASSP, CH2561-9, 1435-1438.

[5] Grossmann.A; Holschneider Kronland-Martinet.R and Morlet,J (1987). Detection of abrupt Changes in sound signal with the help of the wavelet transform. In: Inverse problemes: An interdisciplinary study. Advances in Electronics and Electron physics. Supplement 19 [New York, Academic], 298-306.

[6] Obaidat.M.S. Phonocardiogram signal analysis: techniques and performance comparison. Journal of Medical Engineering \& technologie, vol 17, No 6 (November-December 1993), 221-227.

[7] Boashas, B (1993). Time-frequency signal analysis. In advances in spectrum Estimation, edited by S.Haykin, (NJ: Prentice-Hall).

[8] William J.Williams (1997). Time-frequency and wavelets in Biomedical Signal Processing. Edited by Metin Akay. IEEE Press Serie in BME. 3-8.

[9] Olivier.R and Duhamel.P. Fast algorithms for Discrete and Continuous Wavelet Trnsforms. IEEE Transactions Information Theory, vol38, No.2, (march 1992), 569586

[10] Harris, A., Sutton, G. And Towers, M. (1976). Physiological and clinical aspects of cardiac auscultation. Medicine Ltd, London, Uk.

[11] Y.Meyer (1990). Ondelettes et Opérateurs. Tome1. Paris: Hermann.

[12] S.Mallat (1989). A theory for multiresolution signals decomposition:

The wavelet representation. IEEE Trans.Pattern Anal.Machine Intell, vol 11, pp 674-693.

[13] P.I.J. Keeton, F.S. Schlindwein, Application of Wavelets in Doppler Ultrasound, MCB University Press, Emerald Sensor Review, Vol. 17(1) 1997, pp. 38-45.

[14] S.M Debbal and F.Bereksi-Reguig. The Fast Fourier and the wavelet transforms analysis of the cardiacs sound. Physical and Chemical News (PCN).vol 15, pp 54-59, 2004.

[15] S M Debbal - F Bereksi Reguig: "The fast Fourier transform and the continuous wavelet transform analysis of the phonocardiogram signal "; Journal of Mechanics in Medicine and Biology (JMMB); ISSN: 0219-5194; vol.4, No 3, September 2004 pp 257-272.

[16] Bentley P.M, Grant A, Mc Donnel JTE. Time-frequency and Time-scale for the classification of native and Bioprosthetic valve sounds. IEEE Trans. Biomed. Eng. 45(1), pp 125-128, 1998.

[17] Djebbari.A (1999). Synthèse des méthodes d'analyse temporelle, spectrale et spectrotemporelle du signal phonocardiogramme. Electronic magister thesis of signals and systems. Departement of electronics, faculty of science engineerig, university Aboubekr belkaid Tlemcen, Algeria, 18-24 


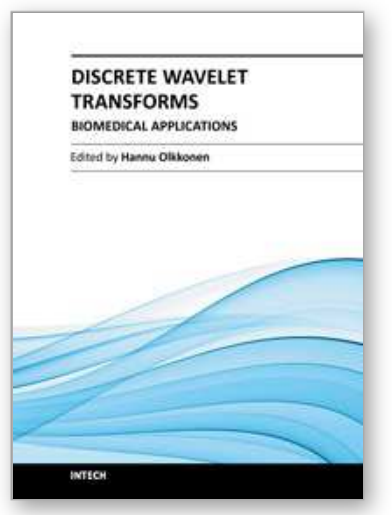

\author{
Discrete Wavelet Transforms - Biomedical Applications \\ Edited by Prof. Hannu Olkkonen
}

ISBN 978-953-307-654-6

Hard cover, 366 pages

Publisher InTech

Published online 12, September, 2011

Published in print edition September, 2011

The discrete wavelet transform (DWT) algorithms have a firm position in processing of signals in several areas of research and industry. As DWT provides both octave-scale frequency and spatial timing of the analyzed signal, it is constantly used to solve and treat more and more advanced problems. The present book: Discrete Wavelet Transforms - Biomedical Applications reviews the recent progress in discrete wavelet transform algorithms and applications. The book reviews the recent progress in DWT algorithms for biomedical applications. The book covers a wide range of architectures (e.g. lifting, shift invariance, multi-scale analysis) for constructing DWTs. The book chapters are organized into four major parts. Part I describes the progress in implementations of the DWT algorithms in biomedical signal analysis. Applications include compression and filtering of biomedical signals, DWT based selection of salient EEG frequency band, shift invariant DWTs for multiscale analysis and DWT assisted heart sound analysis. Part II addresses speech analysis, modeling and understanding of speech and speaker recognition. Part III focuses biosensor applications such as calibration of enzymatic sensors, multiscale analysis of wireless capsule endoscopy recordings, DWT assisted electronic nose analysis and optical fibre sensor analyses. Finally, Part IV describes DWT algorithms for tools in identification and diagnostics: identification based on hand geometry, identification of species groupings, object detection and tracking, DWT signatures and diagnostics for assessment of ICU agitation-sedation controllers and DWT based diagnostics of power transformers. The chapters of the present book consist of both tutorial and highly advanced material. Therefore, the book is intended to be a reference text for graduate students and researchers to obtain state-of-the-art knowledge on specific applications.

\title{
How to reference
}

In order to correctly reference this scholarly work, feel free to copy and paste the following:

S.M. Debbal (2011). Computerized Heart Sounds Analysis, Discrete Wavelet Transforms - Biomedical Applications, Prof. Hannu Olkkonen (Ed.), ISBN: 978-953-307-654-6, InTech, Available from: http://www.intechopen.com/books/discrete-wavelet-transforms-biomedical-applications/computerized-heartsounds-analysis

\section{INTECH}

open science | open minds

\author{
InTech Europe \\ University Campus STeP Ri \\ Slavka Krautzeka 83/A
}

\section{InTech China}

Unit 405, Office Block, Hotel Equatorial Shanghai

No.65, Yan An Road (West), Shanghai, 200040, China 
51000 Rijeka, Croatia

Phone: +385 (51) 770447

Fax: +385 (51) 686166

www.intechopen.com
中国上海市延安西路65号上海国际贵都大饭店办公楼 405 单元 Phone: +86-21-62489820

Fax: +86-21-62489821 
(C) 2011 The Author(s). Licensee IntechOpen. This chapter is distributed under the terms of the Creative Commons Attribution-NonCommercialShareAlike-3.0 License, which permits use, distribution and reproduction for non-commercial purposes, provided the original is properly cited and derivative works building on this content are distributed under the same license. 